Working Paper/Document de travail 2012-41

\title{
Estimating the Policy Rule from Money Market Rates when Target Rate Changes Are Lumpy
}

by Jean-Sébastien Fontaine 
Bank of Canada Working Paper 2012-41

December 2012

\title{
Estimating the Policy Rule from Money Market Rates when Target Rate Changes Are Lumpy
}

\author{
by \\ Jean-Sébastien Fontaine \\ Financial Markets Department \\ Bank of Canada \\ Ottawa, Ontario, Canada K1A 0G9 \\ jsfontaine@bankofcanada.ca
}

Bank of Canada working papers are theoretical or empirical works-in-progress on subjects in economics and finance. The views expressed in this paper are those of the author.

No responsibility for them should be attributed to the Bank of Canada. 


\section{Acknowledgements}

I thank Greg Bauer, Jagjit Chadha, Hans Dewachter, Antonio Diez, Bruno Feunou, Rodrigo Guimares, Jame Hamilton, Scott Hendry, Sean Holly, Glenn Rudebusch, Peter Smith, and participants at the CIMF/IESEG conference "The Yield Curve and New Developments in Macro-finance” for their comments and suggestions. 


\begin{abstract}
Most central banks effect changes to their target or policy rate in discrete increments (e.g., multiples of $0.25 \%$ ) following public announcements on scheduled dates. Still, for most applications, researchers rely on the assumption that the policy rate changes linearly with economic conditions and they do not distinguish between dates with and without scheduled announcements. This assumption is not innocuous when estimating the policy rule based on daily frequency. For the 1994-2011 period, and using an otherwise standard term structure model, I find that accounting for discrete changes leads to economically different estimates. Only the model based on discrete changes depicts a picture that is consistent with existing evidence on the monetary policy rule and risk premium. I study the information content of key policy announcements in the period from the end of 2008, where the policy rate reached a lower bound in the US, until the end of 2011.
\end{abstract}

JEL classification: E43, E44, E47, G12, G13

Bank classification: Asset pricing; Financial markets; Interest rates

\title{
Résumé
}

Le taux cible ou directeur de la majorité des banques centrales fait l'objet de modifications discrètes plutôt que continues (par multiples de 25 points de base par exemple), annoncées publiquement à des dates préétablies. La plupart du temps, toutefois, les chercheurs partent de l'hypothèse que le taux directeur varie linéairement avec les conditions économiques, sans établir de distinction entre les dates d'annonce prévues au calendrier et les autres jours. Cette hypothèse n'est pas sans conséquence lorsque la règle de politique monétaire est estimée à partir de données quotidiennes. Pour la période 1994-2011, et dans le cadre d'un modèle de structure par terme par ailleurs standard, l'auteur montre que la prise en compte du caractère discret des changements du taux directeur aboutit à des estimations différentes du point de vue économique. Le modèle intégrant des changements discrets est le seul dont les résultats cadrent avec ceux existant quant à la règle de politique monétaire et à la prime de risque. L'auteur étudie aussi le contenu informatif des principales annonces effectuées par la Réserve fédérale entre la fin de 2008, où le taux directeur est tombé à un plancher aux États-Unis, et la fin de 2011.

Classification JEL : E43, E44, E47, G12, G13

Classification de la Banque : Évaluation des actifs; Marchés financiers; Taux d'intérêt 


\section{Introduction}

Many central banks change their target (or policy) rates via discrete increments, typically multiples of $0.25 \%$. Moreover, these changes almost always occur following scheduled meetings and the schedule of meetings is known well in advance to market participants. The intent behind this operational procedure is, or is understood to be, to increase the transparency surrounding the central bank's actions and to reduce unnecessary variations in interest rates. Presumably, transparency increases the predictability of future policy rates, tightens the relationship of short-term yields to policy decisions and enhances the effectiveness of the monetary policy transmission mechanism. Indeed, a large literature has shown that short-term interest rates and, in the case of the US, Fed funds futures rates, provide the best forecasts of future target rates and can be used to estimate monetary policy shocks.

An accurate description of expectations as measured from short-term interest rates is crucial for policy makers and market participants. In the following, I compare dynamic term structure models for short-term interest rates that take into account the known schedule of policy meetings. In a first set of results, I compare the implications from a model based on a discrete support, consistent with the fact that target changes take discrete increments, to the implications from the standard Gaussian approximation. ${ }^{1}$ The sample is daily and runs until the end of 2011 . A second set of results analyzes the information content of futures rates around key policy announcements and, more generally, throughout the period where the target reached its lower bound in the US. ${ }^{2}$

Specifically, I ask the following questions. First, does the Gaussian approximation to discrete changes affect policy rule estimates? The answer is yes. Maximum Likelihood estimates based on the discrete distribution are substantially different from Quasi Maximum Likelihood estimates based on the Gaussian distribution. Target rate innovations are far from normal, and in particular, exhibit significant conditional skewness. Second, does the Gaussian approximation affect the estimation of the term structure model? Again, the answer yes. Unsurprisingly, using futures data alters the estimates of the policy rule substantially for each model. More importantly, the estimates from the discrete-support model are consistent with others found in the literature (e.g., Clarida, Galí, and Gertler 2000). The discrete-support model implies substantially more mean-reversion in the target rate than estimates from the Gaussian model. The role of the lagged target rate for future target rates is less important and, consequently, the role of the other variables is more important in the discrete-support model. Moreover, consistent with theory and existing evidence, the discrete-support model produces estimates of the price of macro risk that remain positive and

\footnotetext{
${ }^{1}$ Neglecting the schedule of meetings leads to grossly misspecified models. I do not discuss this case.

${ }^{2}$ The lower bound is typically considered to be zero but the effective lower bound has been $0.25 \%$ so far in the US.
} 
evolve smoothly over time. In contrast, using a Gaussian approximation yields unreasonable risk premium variations.

In a third exercise, I measure and summarize the effect of monetary policy announcements (e.g., press releases following FOMC meetings) on interest rates between the end of 2008, where the target rate reached its lower bound, until the end of 2011. The model is estimated using daily data. I find that every announcement led to a decline in the macro factor. In the model, this translates in a lower risk premium and in lower expected future rates. However, the proximity to the lower bound causes variations in the futures rates to be subdued. This introduces an asymmetry in the information content of the macro factor. It still captures improvements in perceived economic conditions but does not capture further deteriorations. This chapter ends with a discussion of ongoing developments in the literature to address the challenge of the lower bound for existing models of short-term interest rates.

Each model takes into account the known meeting schedule. Each uses the current target rate and a latent macro indicator to drive policy decisions. In each case, the policy rule is linear and variance parameters are constant, with different parameters for days with or without a scheduled policy meeting. In fact the different specifications are identical except for one aspect: the distribution of target rate innovations. The benchmark uses the Gaussian distribution to model target changes. In the alternative models, target rate changes have a discrete distribution, as in the data. In particular, the discrete distribution has fat tails and time-varying skewness. In a first set of comparisons, I impose that the price of risk associated with unexpected target changes is zero. Otherwise, a non-Gaussian model has more flexibility to fit futures data relative to a Gaussian model. I also estimate a variant of the model where the (constant) price of target rate risk is unrestricted. This parameter is significant statistically and economically. Economically, the constant price of target risk induces substantial risk premium variations as well as time-varying volatility in yields.

The existing asset pricing literature focuses on long-term yields or has mostly ignored the facts that target changes take discrete increments and typically occur on known dates. ${ }^{3} \mathrm{~A}$ substantial econometric literature has studied Federal Funds futures and document their ability to predict future target changes. ${ }^{4}$ However, the evidence suggests that the risk premium in Fed funds futures rates exhibits significant variations. ${ }^{5}$ This calls for a dynamic term structure model that combines

\footnotetext{
${ }^{3}$ Notable exceptions include Rudebusch (1995), Balduzzi et al. (1997), Hamilton and Jordà (2002), Piazzesi (2005a) and, recently, Fontaine (2011), Feunou and Fontaine (2012) and Renne (2012).

${ }^{4}$ See Krueger and Kuttner (1996), Cochrane and Piazzesi (2002), Gurkaynak et al. (2007), Ferrero and Nobili (2008), and Hamilton (2009). See also Bhundia and Chadha (1998) for the UK, and Diez de los Rios and Reid (2008) for Canada.

${ }^{5}$ See Sack (2004), Piazzesi and Swanson (2006), and Hamilton and Okimoto (2010)
} 
the information in the cross-section of interest rates and sweeps away the effect of risk premium variations on measures of expectations.

Although it is not the objective of this paper, a target rate specification that accounts for the schedule of policy meeting and for the lumpy changes is applicable in the context of a structural VAR. In particular, one can use the occurrence of policy meetings to obtain a high-frequency identification scheme of monetary policy shocks (Cochrane and Piazzesi, 2002). In addition, one can also use the difference in variance between days with and without meetings to identify the structural shocks (Sentana and Fiorentini 2001, Rigobon 2003). The common identification assumption that monetary policy shocks do not affect macro-economic variables contemporaneously can be implemented easily. I discuss these issues briefly.

Section II introduces the model of the target rate. Section III introduces the corresponding models of the term structure. Section IV discusses the data and the estimation method. Section V presents the estimation results. Section VI studies the futures market from the end of 2008 until the end of 2011. Section VII concludes.

\section{Modeling Short-Term Interest Rates}

\section{A Policy Rules in Practice}

The policy rule followed by the monetary authority to set its target interest rate, $r_{t}$, can be generically defined by,

$$
r_{t+1}=r_{t}+\delta_{r}\left(r_{t}-\bar{r}\right)+\delta_{z}^{\prime}\left(z_{t+1}-\bar{z}\right)+\epsilon_{t+1}^{r}
$$

for a given set of state variables, $z_{t}$, where $\bar{r}$ is interpreted as the natural interest rate, and where $\epsilon_{t+1}^{r}=r_{t+1}-E_{t}\left[r_{t+1}\right]$. The expectation is taken using the information set of market participants or that of the econometrician so that $\epsilon_{t+1}^{r}$ is the unanticipated target change. Equation 1 is often interpreted as the representation of an abstract policy rule. For instance, the celebrated Taylor rule (Taylor, 1993) relates the target Federal Funds rate in the US to deviations of inflation and real GDP from some targets. This is not to say that actual policy decisions follow Equation 1. In Taylor's own words, we should "preserve the concept of a policy rule even in an environment where it is practically impossible to follow mechanically the algebraic formulas economists write down to describe their preferred policy rules." Indeed, the typical central bank does not change its target policy rate mechanically with every change in the state of the economy. Most central banks have instead elected to change their targets only infrequently and then, to use a coarse grid when choosing a new target (i.e. with intervals of $25 \mathrm{bps}$ ). Figure 1 contrasts the evolution of 
the target overnight Federal Funds rate and the 6-month Federal Funds futures rate. Changes to the target rate are lumped together and the evolution of the target rate follows a step function. These properties of target changes have significant implications for the distribution of $\epsilon_{t+1}^{r}$, which we discuss in the next Section. ${ }^{6}$

A central assumption of a typical interest rate model is that the short rate, and yields, evolves smoothly over a continuous support. Therefore, most policy rules studied in the literature do not describe the evolution of the actual policy instrument - the step function in Figure 1 - but instead use a smoother interest rate with a longer maturity. Yields on instruments with very short maturities are often neglected altogether. ${ }^{7}$

The target rate may be a pure jump process but, nonetheless, the evolution of the futures rate appears smooth to our eyes. ${ }^{8}$ But the data say otherwise: changes in yields are are much larger not as smooth - on days with a policy meeting (see the Appendix). The Gaussian approximation may be sufficiently accurate for many research questions related to the evolution of long-term yields. But it is costly in other applications. The approximation affects estimation of the monetary policy rule and of monetary shocks from short term money market instruments (Rudebusch 1995, Cochrane and Piazzesi 2002, Hamilton and Jordà 2002). In turn, different policy rule estimates lead to different measures of the risk premium. ${ }^{9}$ More generally, the standard linear Gaussian approximation creates a tension between capturing the evolution of long-term yields and estimating the policy rule at relatively high frequency (i.e., daily). Indeed, models estimated on yields with long maturities perform poorly for yields with short maturities and imply non-realistic dynamics for the policy rate (see Piazzesi (2005a)).

\section{$B$ Target rate models}

This Section introduces a representation of the policy rule based on the distribution of policy shocks and the timing of policy decisions to address the empirical features of target rate changes. I consider a discrete-time model where the sampling frequency (e.g., daily or weekly), is faster than

\footnotetext{
${ }^{6}$ The European Central Bank, the Bank of Canada, the Bank of England, the Bank of Japan, the Federal Reserve, the Reserve Bank of Australia, the Reserve Bank of New Zealand, among others, all use an overnight rate as policy instrument. In each case, changes are multiples of 25 bps (including 0), and are announced publicly on scheduled announcement dates or follow scheduled policy meetings.

${ }^{7}$ Liquidity effects are also often cited when excluding short-term Treasury bills from estimation (see e.g., Duffee (1996)).

${ }^{8}$ See Appendix A. Futures and interest rates correspond to risk-neutral expectations over all possible realizations of futures target rates. The probability associated with any of these possible realization and, a fortiori, the corresponding expectation can evolve smoothly as new information arrives.

${ }^{9}$ See Equation 24 in the Appendix.
} 
the frequency of scheduled meetings. The target for the overnight rate is given by

$$
r_{t+1}-r_{t} \mid r_{t}, z_{t+1}, I_{t+1} \sim \mathcal{D}\left(\mu\left(r_{t}, z_{t+1}, I_{t+1}\right), \sigma^{2}\left(r_{t}, z_{t+1}, I_{t+1}\right)\right)
$$

for some parametric distribution family, $\mathcal{D}$, conditional on the state variable $z_{t+1}$. The indicator function $I_{t+1}$ is equal to 1 if a policy meeting is scheduled to occur at date $t+1$ and zero otherwise. Therefore, the conditional mean and conditional variance $\mu(\cdot)$ and $\sigma(\cdot)$ can depend on calendar time via the schedule of policy meetings. The policy rate is driven by its own shocks, $\epsilon_{t+1}^{r}$ and by the state variables, $z_{t+1}$. In the following, I contrast models based where the support of the distribution, $\mathcal{D}$, is continuous, $n_{t} \in \mathbb{R}$, or discrete, $n_{t} \in(\ldots,-2,-1,0,1,2, \ldots)$.

\section{Model I : Normal-Markov}

The standard linear-Gaussian policy rule approximation is nested in Equation 2 but where the mean function, $\mu(\cdot)$, does not depend on calendar time and where the variance is constant. I consider the more general alternative where the schedule of policy meetings affects the distribution of policy shocks. Specifically, the specification of the conditional mean $\mu(\cdot)$ different for days that followed by a scheduled meeting (i.e., $I_{t+1}=1$ ) than for days that are not followed by a scheduled meeting (i.e., , $\left.I_{t+1}=0\right)$. Hence, we have two cases :

$$
\begin{aligned}
r_{t+1}-r_{t} \mid r_{t}, z_{t+1},\left(I_{t+1}=0\right) & \sim \mathcal{N}\left(0, \sigma_{0}^{2}\right) \\
r_{t+1}-r_{t} \mid r_{t}, z_{t+1},\left(I_{t+1}=1\right) & \sim \mathcal{N}\left(\delta_{r}\left(r_{t}-\bar{r}\right)+\delta_{z}^{\prime}\left(z_{t+1}-\bar{z}\right), \sigma_{1}^{2}\right) .
\end{aligned}
$$

Following Piazzesi (2005a) and Fontaine (2011), the expected target change is state-dependent on days with a scheduled policy meeting but zero otherwise. The variance differs between days with or without a scheduled meeting but it is constant otherwise.

\section{Model II : Skellam-Homoscedastic}

Next, I follow Fontaine (2011) who uses the Skellam distribution (Skellam, 1946) to confine target rate changes to a discrete support. Heuristically, this distribution corresponds to the difference between two Poisson random variables. If $n_{t+1}^{u}$ and $n_{t+1}^{d}$ have independent conditional Poisson distributions with parameters $\lambda^{u}\left(r_{t}, z_{t+1}, I_{t+1}\right)$ and $\lambda^{d}\left(r_{t}, z_{t+1}, I_{t+1}\right)$, respectively, then $n_{t+1}=n_{t+1}^{u}-n_{t+1}^{d}$ has a Skellam distribution, $\mathcal{S} \mathcal{K}$. Then, discrete target changes can be written as,

$$
r_{t+1}-r_{t}=\Delta n_{t+1}=\Delta\left(n_{t+1}^{u}-n_{t+1}^{d}\right),
$$

where $\Delta=0.25 \%$ is known. I assume that the intensity parameters on days without a scheduled 
meeting are given by,

$$
\lambda^{u}\left(r_{t}, z_{t+1}, 0\right)=\lambda_{0} \quad \lambda^{d}\left(r_{t}, z_{t+1}, 0\right)=\lambda_{0}
$$

to allow for target rate change following unscheduled FOMC meetings, and by

$$
\begin{aligned}
& \lambda^{u}\left(r_{t}, z_{t+1}, 1\right)=\lambda_{1}+\lambda_{r}\left(r_{t}-\bar{r}\right)+\lambda_{z}\left(z_{t+1}-\bar{z}\right) \\
& \lambda^{d}\left(r_{t}, z_{t+1}, 1\right)=\lambda_{1}+\lambda_{r}\left(r_{t}-\bar{r}\right)+\lambda_{z}\left(z_{t+1}-\bar{z}\right),
\end{aligned}
$$

when there is a meeting. Then, target changes are characterized by

$$
\begin{aligned}
r_{t+1}-r_{t} \mid r_{t}, z_{t+1},\left(I_{t+1}=0\right) & \sim \mathcal{S K}\left(0, \sigma_{0}^{2}\right), \\
r_{t+1}-r_{t} \mid r_{t}, z_{t+1},\left(I_{t+1}=1\right) & \sim \mathcal{S K}\left(\delta_{r}\left(r_{t}-\bar{r}\right)+\delta_{z}\left(z_{t+1}-\bar{z}\right), \sigma_{1}^{2}\right),
\end{aligned}
$$

with

$$
\begin{array}{cc}
\sigma_{0}^{2}=2 \lambda_{0} \Delta^{2} & \sigma_{1}^{2}=2 \lambda_{1} \Delta^{2} \\
\delta_{r}=2 \lambda_{r} \Delta & \delta_{z}=2 \lambda_{z} \Delta
\end{array}
$$

Model I and Model II have linear mean function, constant variance, the same number of parameters and can be compared directly. The only difference between Equations 3 and 6 is the distribution of policy innovations. The Skellam distribution imposes that the probability of observing a target change which is not a multiple of $0.25 \%$ is zero. Moreover, this distribution inherits features of the Poisson distribution. Importantly, it allows for time-varying asymmetry (i.e., skewness) in policy innovations, given by

$$
\operatorname{Skew}\left[r_{t+1}-r_{t} \mid r_{t}, z_{t+1},\left(I_{t+1}=1\right)\right]=\frac{1}{\sigma_{1}}\left(\delta_{r}\left(r_{t}-\bar{r}\right)+\delta_{z}\left(z_{t+1}-\bar{z}\right)\right) .
$$

In other words, the asymmetry in the distribution of policy innovations is proportional to the mean. The shape of the distribution of target rates also changes when its mean changes. For instance, following an increase in the expected target changes, the probability of specific target hikes (e.g., $0.25 \%, 0.50 \%$ ) increases more than the probability of the corresponding target cuts with the same magnitude (e.g., $-0.25 \%,-0.50 \%)$. The Skellam distribution also has fat tails. Its excess kurtosis is inversely proportional to the variance,

$$
\operatorname{Kurt}\left[r_{t+1}-r_{t}\right]=3+\frac{\Delta^{2}}{\sigma^{2}} .
$$




\section{Term Structure Models}

This Section specifies the dynamics of $z_{t}$ and of the pricing kernel to complete the term structure models. These additional assumptions are identical across short rate specifications and remain unchanged throughout the empirical investigation below. Note that all the models considered below belong to the family of 2-factor dynamic affine term structure models (Piazzesi, 2005b).

\section{A State Variables}

In the spirit of standard macro-finance models, I assume the state-vector, $z_{t+1}$, has a stationary auto-regressive representation,

$$
z_{t+1}-\bar{z}=\phi\left(z_{t}-\bar{z}\right)+\epsilon_{t+1}
$$

where $\epsilon_{t+1} \sim N\left(0, \sigma_{z}^{2}\right)$. As in Piazzesi (2005a) and Fontaine (2011), this latent process is intended to capture variations in the economy relevant to the FOMC. Next, I introduce the spread between the target overnight rate and the effective market rate, $s_{t}=\tilde{r}_{t}-r_{t}$. The effective spread is included for completeness since observed yields and Federal Funds futures depend on the realization of the market overnight rate (see below). In practice, however, $s_{t}$ plays no significant role in expectations (Balduzzi et al., 1997) since it exhibits little persistence. In addition, it does not affect the evolution of $r_{t}$ or other macro variables. ${ }^{10}$ The target spread $s_{t}$ follows a leptokurtic process,

$$
s_{t+1}-\bar{s}=\phi_{s}\left(s_{t}-\bar{s}\right)+\epsilon_{t+1}^{s}+J_{t+1}^{s}
$$

where $\epsilon_{t}^{s}$ is i.i.d with distribution $N\left(0, \sigma_{s}\right)$ and $J_{t+1}^{s}$ is i.i.d. compound Poisson with number of jumps $n_{t+1}^{s} \sim P\left(\lambda_{s}^{J}\right)$ and jump size $\nu_{t+1}^{s} \sim N\left(\nu_{s}, \omega_{s}^{2}\right)$, conditional on the number of jumps. The asymmetry and fat tails of the target spread are well documented and large shocks typically occur around the maintenance period for required reserve (e.g., Hamilton 1996, Piazzesi 2005a).

To summarize, the state vector $X_{t}=\left[r_{t} z_{t} s_{t}\right]$ combines the target rate, a latent macro factor and the effective spread. Equations 1011 can be combined with any of the short-rate specifications above into an auto-regressive representation for $X_{t}$ (i.e., the mean functions are all linear.),

$$
X_{t+1}-\bar{X}=\phi\left(I_{t+1}\right)\left(X_{t}-\bar{X}\right)+\eta_{t+1},
$$

where the innovations $\eta_{t+1}$ are independent through time, with $E_{t}\left[\eta_{t+1}\right]=0$. The parameters corresponding to the short-rate rule - the first line of the matrix $\Phi(\cdot)$ - change with $I_{t+1}$. Equation 12

\footnotetext{
${ }^{10}$ The evidence of a liquidity effect between reserves and macro variables is weak and subject to controversial identification assumptions (Hamilton, 1997). Thornton (2006) discusses the relation between reserve (supply) management operations by the Fed, reserve (demand) forecast errors by the Fed, and the effective overnight Federal Funds rate.
} 
corresponds to the state equation in the state-space system below.

The auto-regressive representation implies that the policy response function is applicable in the context of a structural VAR. In particular, one can use the occurrence of policy meetings to obtain a high-frequency identification scheme of monetary policy shocks (Cochrane and Piazzesi, 2002). In addition, one can also use the difference in variance between days with and without meetings to identify the structural shocks (Sentana and Fiorentini 2001, Rigobon 2003). A common assumption to identify the structural shock is that monetary policy shocks do not affect macroeconomic variables contemporaneously. This can be easily implemented here. Moreover, innovations in any of the state variables affect $r_{t+1}$ on FMOC days only via the policy rule parameters. ${ }^{11}$

\section{$B \quad$ Pricing kernel}

I consider a standard exponential-affine kernel, $M_{t+1}$,

$$
M_{t+1}=\frac{\exp \left(\lambda_{s} \epsilon_{t+1}^{s}+\lambda_{t, z} \epsilon_{t+1}^{z}\right)}{E_{t}\left[\exp \left(\lambda_{s} \epsilon_{t+1}^{s}+\lambda_{t, z} \epsilon_{t+1}^{z}\right)\right]} .
$$

where $\lambda_{s}$ is the price of risk associated with innovation in the effective spread $s_{t}$ and $\lambda_{t, z}$ is the price of risk associated with innovation in the macro factor $z_{t}$. Following Duffee (2002), the price of macro risk is linear,

$$
\lambda_{t, z}=\lambda_{0}+\lambda_{z, r} r_{t}+\lambda_{z, z} z_{t}
$$

This specification embodies several restrictions on the prices of risk. First, and most importantly, the price of risk associated with innovations to $z_{t+1}$ varies with the latent economic indicator, $z_{t}$, and with the current target rate, $r_{t}$. This introduces business cycle variations in the risk premium implicit in futures rates that has been documented by Piazzesi and Swanson (2006). There is no other source of risk premium variations. Second, the price of target rate risk to zero. This implies that the policy rule is the same under the risk-neutral and the historical probability measures. ${ }^{12}$ It also maintains the comparability between different models. Otherwise, the effect of risk on the autoregressive matrix would not be the same whether the short rate is Gaussian or Skellam (see Section C). I relax this assumption in Section C. Finally, the price of risk associated with $\epsilon_{t+1}^{s}$ is constant and induces a constant risk premium. The effective spread is highly volatile and shows no

\footnotetext{
${ }^{11}$ Another way to see the implicit structural restriction is by approximating $\eta_{t+1}$ with $\Sigma\left(I_{t+1}\right) u_{t+1}$ where $u_{t+1}$ are uncorrelated white noises and the matrix $\Sigma\left(I_{t+1}\right)$ is of the form,

$$
\Sigma\left(I_{t+1}\right)=\left[\begin{array}{cc}
\sigma_{r}^{2}\left(I_{t+1}\right) & \sigma_{r, Y}\left(I_{t+1}\right) \\
0 & \Sigma_{z}
\end{array}\right] .
$$

and $\sigma_{r, Y}\left(I_{t+1}\right)$ depends on primitive parameters of $\lambda_{t}^{u}$ and $\lambda_{t+1}^{d}$.

${ }^{12}$ Fontaine (2011) discuss the parameter shifts between the historical and the risk-neutral measures.
} 
persistence and it makes little sense to allow the risk premium to vary accordingly. Overall, these restrictions on the price of risk parameters simplify the interpretation of risk premium variations. Moreover, affine models with unrestricted prices of risk tend to over-fit the data and often produce astronomically high Sharpe ratios (Duffee, 2009).

\section{Estimation}

\section{A Data}

I use a daily sample from February 4th 1994 until the end of 2011. This period covers 144 scheduled meetings. The FOMC effected changes to its target rate following 53 of these scheduled meetings and, also, following 7 unscheduled meetings. The data include the target and effective overnight funds rates, available from the Federal Board of Governors, and Fed Funds futures data, available from the CME. I use contracts with horizons from 1 to 6 months. Contracts with longer horizons are illiquid for most of the sample - days with no transactions on the exchange are a common occurrence. ${ }^{13}$ Table I(a) presents summary statistics of the futures rates. The average term structure of futures rates is upward sloping. Table I(a) presents summary statistics of target rate changes following schedule FOMC meetings as well as for the effective spread $s_{t}$ on all days. Target changes averaged zero with standard deviation close to $25 \mathrm{bps}$ in this sample. There is also some evidence of negative skewness and of kurtosis in the distribution of target rate change consistent with a specification based on the Skellam distribution. The effective spread exhibits a distribution skewed to the right and with substantial fat tails. The estimate of its kurtosis is 32.6 !.

\section{B Measurement Equations}

The measurement equation includes the target rate, which is observed without error. The corresponding conditional log-likelihood is based on one of the Gaussian density or the Skellam probability mass function,

$$
\begin{aligned}
r_{t} \mid r_{t-1}, z_{t} & \sim \mathcal{N}\left(\mu_{I_{t}}\left(r_{t-1}, z_{t}\right), \sigma_{I_{t}}^{2}\right) \\
\text { or } & \\
r_{t} \mid r_{t-1}, z_{t} & \sim \mathcal{S K}\left(\mu_{I_{t}}\left(r_{t-1}, z_{t}\right), \sigma_{I_{t}}^{2}\right) .
\end{aligned}
$$

\footnotetext{
${ }^{13}$ The Fed changed its operational policy starting with the February 1994 meeting and has since announced any target changes following its policy meeting. The history of target rate changes is available at http://www.newyorkfed.org/markets/statistics/dlyrates/fedrate.html
} 


\section{Table I: Summary Statistics}

Panel (a) provides the averages and standard deviations of futures rates for horizons from 1 to 6 months. Panel (b) provides summary statistics for target rate changes following scheduled FOMC meeting and for the spread $s_{t}$ between the effective overnight rate and the target rate (for all days). Statistics include the average, $\mu$, standard deviation, $\sigma$, skewness, kurtosis and auto-correlation of order 1, $\rho(1)$. Annualized basis points. Daily data from Jan. 3rd, 1994 to Dec. 30th, 2011.

(a) Futures rates

\begin{tabular}{lrrrrrr}
\hline \hline Horizon & $\mathbf{1}$ & $\mathbf{2}$ & $\mathbf{3}$ & $\mathbf{4}$ & $\mathbf{5}$ & $\mathbf{6}$ \\
\hline$\mu$ & 3.40 & 3.41 & 3.44 & 3.46 & 3.50 & 3.53 \\
$\sigma$ & 2.17 & 2.18 & 2.19 & 2.20 & 2.20 & 2.21 \\
\hline
\end{tabular}

(b) Overnight rate and effective spread

\begin{tabular}{lrrrrl}
\hline \hline & $\mu$ & $\sigma$ & skew & kurt & $\rho(1)$ \\
\hline$r_{t}-r_{t-1}$ & 0 & 22.5 & -0.5 & 5.2 & 0.55 \\
$s_{t}$ & 1.7 & 18.0 & 0.8 & 32.6 & 0.51 \\
\hline \hline
\end{tabular}

Next, the measurement vector includes futures rates, stacked in vector $F_{t} \cdot{ }^{14} \mathrm{I}$ assume that futures rates are measured with errors, $F(t, n)=F\left(X_{t}, n\right)+\nu_{t}$, where $F\left(X_{t}, n\right)$ is the model-implied futures rate and $\nu_{t}$ is a vector of i.i.d zero-mean uncorrelated Gaussian measurement errors with variance given by

$$
\xi\left(T_{n}\right)^{2}=\left(\xi_{0}+\xi_{1} T_{n}\right)^{2}
$$

where $T_{n}>0$ is the number of days until the end of the reference contract. Hence the log-likelihood of the futures rates is based on the Gaussian density,

$$
F_{t} \mid X_{t} \sim \mathcal{N}\left(F\left(X_{t}\right), \xi^{2}\right)
$$

The model-implied formula for futures rate can be derived from its payoff. With no loss of generality, I standardize the notional of the contract to 1. A futures contract settles at the end of a reference calendar month, $n$. The Federal Funds futures contract is essentially a swap between a variable rate and a fixed rate. The variable leg is the average overnight Fed Funds rate in the reference calendar month, $\bar{r}_{n}$. With no cash exchange at inception, the fixed rate of the contract,

\footnotetext{
${ }^{14}$ The quoted price of this contract, $P(t, n)$, is given by $P(t, n)=100-F(t, n) \times 3600$. Due to weekends or holidays, the settlement date may not coincide with the last day of the month. For simplicity, this possibility is not visible in Equation 18 but I use CME's "Following Business Days" in the following.
} 
$F(t, n)$, must equal to expectation of average overnight rate under the risk-neutral measure,

$$
\begin{aligned}
F(t, n)=E_{t}\left[M_{t, t+T_{n}} \bar{r}_{n}\right] & =E_{t}\left[M_{t, t+T_{n}} D_{n}^{-1} \sum_{i=T_{n}-D_{n}}^{T_{n}} r_{t+i}\right] \\
& =D_{n}^{-1} \sum_{i=T_{n}-D_{n}}^{T_{n}} E_{t}\left[M_{t, t+T} r_{t+i}\right] \\
& =D_{n}^{-1} \sum_{i=T_{n}-D_{n}}^{T_{n}} f\left(t, i, T_{n}\right),
\end{aligned}
$$

where $T_{n}$ is the number of days between $t$ and the end of the reference month and $D_{n}$ is the number of days in that month. In turn, the rate on a singleton futures contract, $f(t, h, T)$, for the reference day $t+h$ and settling at date $t+T$, with $0 \leq h \leq T$, is given by

$$
\begin{gathered}
\left.f(t, h, T)=\exp \left(d_{0}(t+h, T-h)+c_{0}\left(u^{*}, t, h\right)\right)+c\left(u^{*}, t, h\right)^{\prime} X_{t}\right) \\
\times\left[c_{0}^{\prime}\left(u^{*}, t, h\right) C_{r}+X_{t}^{\prime} c^{\prime}\left(u^{*}, t, h\right) C_{r}\right],
\end{gathered}
$$

where $u^{*}=d(t+h, T-h)$ and $C_{r}=\left[\begin{array}{lll}1 & 0 & 0\end{array}\right]^{\prime}$. Note that the coefficient recursions have two dimensions. They depend on the maturity, $h$, and also on calendar time, $t$, via the schedule of policy meetings. See Fontaine (2011) for the derivation of futures rates and the computation of the corresponding coefficient recursions.

\section{Filter and Likelihood}

Equation 12 provides the transition equation of the state-space system. However, $z_{t}$ is not observed and must be filtered from the data. I use the Unscented Kalman Filter [UKF] since the measurement equation of futures rates is not linear in $X_{t}$ (see Equation 19). The UKF is an approximate filter that matches the first two moments of the state distribution. A QML estimator is feasible but the variance in the log-likelihood of the $F_{t}$ and $r_{t}$ must be adjusted for the uncertainty associated with the filter for $z_{t} \cdot{ }^{15}$ Conditional on values of the latent state variables, the joint

\footnotetext{
${ }^{15}$ This adjustment is standard in linear models and exact when the density is Gaussian (see e.g. Hamilton, 1994). However, the measurement equation is not linear (but Gaussian) in the case of futures and it is not Gaussian (but linear) in the case of the target rate. See Fontaine (2011) for details.
} 
(quasi) log-likelihood is given by

$$
\begin{aligned}
L(\Theta ; Y) & =\sum_{t=1}^{T} \log \left(f_{t}\left(\tilde{Y}_{t} \mid \tilde{Y}_{\underline{t-1}}, I_{t} ; \theta\right)\right) \\
& =\sum_{t=1}^{T} \log \left(f\left(Y_{t} \mid \underline{\tilde{Y}_{t-1}}, r_{t}, s_{t}, I_{t}\right) f\left(r_{t} \mid \tilde{Y}_{\underline{t-1}}, I_{t}\right) f\left(s_{t} \mid s_{t-1}\right)\right)
\end{aligned}
$$

where all model parameters are grouped in the vector $\Theta$, and $\tilde{Y}_{\underline{t}}$ summarizes the history of observable variables for $t=1, \ldots, t$. The conditional likelihood of $Y_{t}$ depends on $t$ through the deterministic FOMC schedule. I use the following assumptions to identify the location, the scale and the sign of the latent factor, $z_{t}: \bar{z}=0, \sigma_{z}=1 / 360 \mathrm{bps}$ and $\delta_{z} \geq 0$. These are standard in the term structure. In practice, these assumptions imply that the latent indicator is centered around zero, that it has the same unit than a daily interest rate, and that an increase in $z_{t}$ is associated with an increase of the expected target rate and should corresponds to improving economic conditions. Finally, we have that $\left|\phi_{z}\right|<1$ and $-2<\left|\delta_{r}\right|<0$ so that $z_{t}$ and the target rate are stationary.

\section{Results}

\section{A Effective Spread}

The restrictions above imply the separation of the marginal likelihood of $s_{t}$ and, therefore, its dynamics can be estimated separately. Table II presents maximum likelihood estimates of Equation 11. Panel (a) presents the estimates based on data until Dec. 15th 2008 when the FOMC set the interest rates on required reserve balances and on excess balances at 25 basis points. I present results based on the earlier sample separately to document the change in the behavior of the effective spread since the Fed scaled up its liquidity intervention and started paying interest on reserves in the fall of 2008 to implement its policy rate target. Bech and Klee (2009) discusses the changes to the Federal Reserve's operation framework. Figure 2 makes evident the change in behavior in 2008. Persistent downward pressures on the effective spread started early in the fall of 2008. The Fed started paying interest on reserves in early October 2008. The results are similar for different starting dates. Panel (b) presents the estimates based on all the data until Dec. 30th 2011.

All parameters are precisely estimated. The effective spread exhibits little persistence and its unconditional mean is close to zero in the first sample. This reflects the Fed's ability to counter-act predictable deviation of the market rate from its target. The innovations to the effective spread 
have an asymmetric distribution with fat tails. The estimate of the jump intensity parameter, $\lambda_{s}^{J}$, is 0.25 . Innovations to the effective spread mix two Gaussian distributions on average 1 day out of four. When no jump occurs, the distribution is Gaussian with zero mean and with a volatility of 5bps. Otherwise, the distribution is mixed with another Gaussian distribution with a mean of 2 bps and a much wider volatility of 30 bps. When we include data after 2008, the innovation distribution remains broadly unchanged. However, the effective spread becomes more persistent and its mean becomes more negative. In other words, deviations of the market rate are more negative and more persistent in the later part of the sample. This reflects the Fed's emphasis on the provision of liquidity and, later, the effect of quantitative easing. The Fed now rely on interest paid on reserves to maintain the market rate close to its target (Bech and Klee, 2009).

\section{Table II: Effective Spread Parameters}

Maximum likelihood estimates of dynamics of the effective spread, $s_{t}=\tilde{r}_{t}-r_{t}$

$$
s_{t+1}=\mu_{s}+\phi_{s} s_{t}+\epsilon_{t+1}^{s}+J_{t+1}^{s},
$$

where $s_{t}$ is in annualized percentage, $\epsilon_{t}^{s}$ is i.i.d with distribution $N\left(0, \sigma_{s}\right)$ and $J_{t+1}^{s}$ is i.i.d. compound Poisson with number of jumps $n_{t+1}^{s} \sim P\left(\lambda_{s}^{J}\right)$ and jump size $\nu_{t+1}^{s} \sim N\left(\nu_{s}, \omega_{s}^{2}\right)$, conditional on the number of jumps. Panel (a) displays the estimates in a daily sample from Feb. 3rd, 1994 to Dec. 15th, 2008. Panel (b) displays the estimates in a daily sample from Feb. 3rd, 1994 to Dec. 30th, 2011.

(a) 1994-2008 Sample

\begin{tabular}{rrrrrr}
\hline \hline$\mu_{s}$ & $\phi_{s}$ & $\sigma_{s}$ & $\nu_{s}$ & $\omega_{s}$ & $\lambda_{s}^{J}$ \\
-0.003 & 0.23 & 0.051 & 0.019 & 0.305 & 0.248 \\
$(0.00008)$ & $(0.002)$ & $(0.0005)$ & $(0.0027)$ & $(0.0012)$ & $(0.0028)$ \\
\hline \multicolumn{7}{c}{} \\
\hline \hline$\mu_{s}$ & $(\mathrm{~b}) 1994-2011$ Sample \\
-0.0138 & 0.56 & 0.052 & 0.031 & 0.280 & 0.222 \\
$(0.0004)$ & $(0.011)$ & $(0.002)$ & $(0.002)$ & $(0.07)$ & $(0.011)$ \\
\hline
\end{tabular}

\section{B Policy Rules}

This Section estimate the policy rule equation based on target rate date only - excluding futures rates data. I compare policy rule estimates based on different distributional assumptions for the policy shocks (i.e., Equation 3 and Equation 6). Importantly, the Gaussian policy rule and the discrete policy rule have the same linear conditional mean specification, the same number of parameters and a constant variance. The Gaussian policy rule approximates the distribution of target rate changes with a continuous support over the real line. In contrast, the discrete 
distribution imposes that the target rate can only take discrete values (i.e., multiples of $0.25 \%$ ). The results show that changing the distribution affects the estimates of policy rule coefficients significantly.

Table III presents results for each models. Using a discrete distribution yields a higher likelihood value than using a continuous distribution (1533.3 vs 1504.5). Clearly, these models are not nested and a standard likelihood ratio test is not applicable. Nonetheless, ranking the log-likelihoods remains a consistent basis for model selection (Sin and White, 1996) and, therefore, I conclude that the data favour the Skellam distribution. ${ }^{16}$

\section{Table III: Policy Rule Parameters}

Maximum likelihood (or Quasi maximum likelihood) estimates of the policy rule,

$$
r_{t+1}-r_{t} \mid r_{t}, z_{t+1}, I_{t+1}=1 \sim \mathcal{D}\left(\delta_{r}\left(r_{t}-\bar{r}\right)+\delta_{z}\left(z_{t+1}-\bar{z}\right), \sigma_{1}^{2}\right),
$$

using target rate observations only, excluding futures rates data, on days with FOMC meetings (i.e., $I_{t+1}=1$ ) where $r_{t}$ is the target rate, $z_{t}$ is a latent economic indicator, and $\mathcal{D}(\cdot, \cdot)$ is the distribution of the policy rate target innovations, eta . Panel (a) displays results based on a Gaussian (continuous) distribution for the policy innovation and Panel (b) displays results based on a Skellam (discrete) distribution. The estimate of $\sigma_{1}$ for each model is reported in annualized basis point units. The persistence of $z_{t}$ is fixed at $\phi_{z}=0.995$ in each case. Daily data from Feb. 3rd, 1994 to Dec. 30th, 2011. Standard errors in parenthesis.

(a) Gaussian Innovations

\begin{tabular}{cccc}
\hline \hline$\delta_{r}$ & $\delta_{z}$ & $\sigma_{1}$ & QML \\
-0.014 & 0.031 & 0.161 & 1504.5 \\
$(0.006)$ & $(0.003)$ & $(0.013)$ & \\
\hline
\end{tabular}

(b) Discrete Innovations

\begin{tabular}{cccc}
\hline \hline$\delta_{r}$ & $\delta_{z}$ & $\sigma_{1}$ & ML \\
$-2 \times 10^{-12}$ & 0.006 & 0.208 & 1533.3 \\
$\left(1 \times 10^{-4}\right)$ & $(0.0018)$ & $(0.015)$ & \\
\hline
\end{tabular}

Each model attributes a different role to $z_{t}$. The estimates of the coefficient $\delta_{z}$ is 0.03 in the Gaussian model and 0.006 in the Skellam model. Both estimates are significant (they are nonnegative by construction). Moreover, in the case of the Gaussian model, the target rate plays a significant role in its own mean-reversion, $\hat{\delta}_{r}=-0.014$, but it is insignificant in the discrete model. In fact, the point estimate is trivially small (i.e., $-2 e^{-12}$ ). Therefore, a specification where the lag

\footnotetext{
${ }^{16}$ A poorly specified model with discrete support could be rejected in favour of a model with a continuous support.
} 
target rate plays no role,

$$
r_{t+1}-r_{t} \mid r_{t}, z_{t+1}, I_{t+1}=1 \sim \mathcal{S K}\left(\delta_{z}\left(z_{t+1}-\bar{z}\right), \sigma_{1}^{2}\right)
$$

cannot be rejected on the basis of target rate data only when we use the Skellam distribution but not otherwise. In other words, variations in $z_{t}$ are sufficient. But why are the estimates different? The answer must follow from the only difference between the two models. ${ }^{17}$ In the discrete-support model, the asymmetry in the distribution of target changes is proportional to its mean in the discrete model. In the discrete model, the coefficient $\delta_{z}$ drives variation in the expected target changes. Since this coefficient is positive, improving economic conditions implies that the likelihood of a target hike increases by more than the decrease in the likelihood of a target cut with the same magnitude. In contrast, the likelihood changes are symmetric in the Gaussian case. Then, one way to shift the distribution function is to also include the target rate as a conditioning variable. In this case, similar economic conditions, as measured by $z_{t}$, will be associated with different distribution of future target changes depending on the current value of the target rate.

Finally, target innovations have a relatively large standard deviation in both models. This is not surprising since these simple policy rules do not use any conditioning variable beyond the current target rate and the filtered value of the latent factor, $z_{t}$. In turn, the latent factor is filtered only based on past target rate changes. The objective of this section is not too provide a good fit of target rate changes but to highlight the consequences different distributional assumptions on the coefficient estimates. The following Section uses term structure data as conditioning data.

\section{Term Structure Models}

This Section presents estimation results for the full dynamic term structure model in Section III using futures rates and the target rate data, jointly. Parameters of the effective spread dynamics are fixed at their values estimated in the entire sample (see Section A above). I first compare term structure models based on the Gaussian or the Skellam distribution but where target rate risk is not priced (Model I vs. Model II). These models have identical parametric specification and the same number of parameters. The only difference is the distribution of the policy shocks. Clearly, the results below differ from those in Table III since a much richer information set is used here. The term structure of futures rates brings forward-looking information about the path of future target rates and helps estimate the policy rule reliably.

\footnotetext{
${ }^{17}$ More formally, these estimates were obtained from minimizing different objective functions (i.e., different estimator). There is no reason why these should be equal in a finite sample. In addition, the convergence of the QML estimator based on the Gaussian distribution to the parameters of interest only holds asymptotically and if the distribution is symmetric.
} 


\section{Pricing errors}

Table IV compares the Root Mean Squared pricing Error (RMSE), as well as the estimates of standard deviations, $\xi(n)$. All are reported in annualized basis points. Panel (a) and Panel (b) displays results for the Gaussian model and the discrete model, respectively. Each model provides a good fit of futures rates. ${ }^{18}$ Moreover, these models cannot be distinguished on the basis of the RMSEs. But this should not come as a surprise. Futures rates only depends on the the variance and conditional mean of future target rates. The first two moments have identical parametric specification in each model. The asymmetry in the Skellam distribution does not affect the modelimplied futures rates since target rate risk is not priced.

\section{Table IV: Pricing Errors}

Root Mean Squared pricing Errors (RMSE) and standard deviations of measurement errors $\xi\left(T_{n}\right)$ for futures rates with one to six months to maturity in annualized basis points. Panel IV(a) presents results for the Gaussian model. Panel IV(b) presents results for the Discrete model. Daily data from Feb. 3rd, 1994 to Dec. 30th, 2011.

(a) Pricing Errors - Gaussian Model

\begin{tabular}{ccccccc}
\hline \hline & \multicolumn{7}{c}{ Maturity } \\
\cline { 2 - 7 } & 1 & 2 & 3 & 4 & 5 & 6 \\
RMSE & 4.38 & 6.67 & 4.99 & 3.53 & 2.29 & 4.04 \\
$\xi(n)$ & 5.33 & 4.99 & 4.65 & 4.31 & 3.97 & 3.63 \\
\hline
\end{tabular}

(b) Pricing Errors - Discrete Model

\begin{tabular}{ccccccc}
\hline \hline & \multicolumn{7}{c}{ Maturity } \\
\cline { 2 - 7 } & 1 & 2 & 3 & 4 & 5 & 6 \\
RMSE & 4.43 & 6.65 & 4.92 & 3.58 & 2.26 & 4.12 \\
$\xi(n)$ & 5.28 & 5.06 & 4.84 & 4.62 & 4.40 & 4.18 \\
\hline
\end{tabular}

\section{Parameter estimates}

Table V and Table VI display parameter estimates for the Gaussian and discrete models, respectively. Unsurprisingly, both sets of results imply that the daily evolution of the target rate is very persistent. In fact, it is statistically indistinguishable from a unit root. But the models differ substantially in how they build the persistence into the target rate equation.

\footnotetext{
${ }^{18}$ Piazzesi (2005a) obtains average absolute pricing errors above 7 bps for LIBOR rates with comparable maturities, Feldhtter and Lando (2008) obtains an RMSE of 22 bps for 3-mth LIBOR rates. These author fit a broader set of maturities and of instruments.
} 
Estimates of policy rule parameters from the Gaussian model are $\hat{\delta}_{r} \sim 0$ and $\hat{\phi}_{z}=0.9948$. In contrast, the estimates from the discrete model are $\hat{\delta}_{r}=0.30$ and $\hat{\phi}_{z}=0.9999$. Consider first the persistence of the macro factor. The two point estimates have very different forecasting implications. At a 6 -month horizon we have $0.9948^{180}=0.40$ and $0.9999^{180}=0.98$ (the frequency is daily). Hence, in the Gaussian model, $60 \%$ of $z_{t}$ innovations dissipate within six months relative to only $2 \%$ in the model based on the Skellam distribution. Therefore, the effect of current innovations in $z_{t}$ on future target rate changes is much smaller in the Gaussian model since estimates of the $z_{t}$ coefficients $\delta_{z}$ are similar (0.016 vs 0.023$)$.

Next, consider the estimate of $\delta_{r}$. This measures the degree of mean-reversion in the target rate. ${ }^{19}$ The target is nearly a unit root in the Gaussian model. There is no significant meanreversion when $z_{t}=\bar{z}$. In contrast, the discrete-support model imparts substantial mean-reversion to the target rate. If the macro factor is held at its mean $\left(z_{t}=\bar{z}\right)$ then the target rate is expected to retrace $30 \%$ of the path toward its unconditional mean at each scheduled meeting. Clarida et al. (2000) find estimates of the autocorrelation coefficient on the lag short rate between 0.73 and 0.88 in the post-Volcker era. For comparison, the corresponding coefficient estimate obtained here is $\hat{\rho}=1-0.30=0.70$. These estimates are not directly comparable since they were obtained from a different sample period and using different instruments. Moreover, the estimates from Clarida et al. (2000) correspond to quarterly dynamics while the estimates obtained here correspond to the frequency of scheduled meetings. Nonetheless, this rough comparison suggests that the level of mean-reversion estimated from the Gaussian model is inconsistent with the data (biased) while the estimate from the model based on the Skellam distribution offers a consistent picture.

Looking back at Section B, we see that estimates of $\delta_{r}$ and $\delta_{z}$ in Table III differ substantially from those obtained here. Again, estimates from the full no-arbitrage model use forward-looking information from futures rates. Using futures data changes the description of the policy rule drawn by each model. Moreover, the more realistic model, based on a discrete distribution, appears consistent with existing results and with economic intuition. Estimates from the discrete-support allow for a rapid reversion of the policy rate when economic conditions returns to a normal state. On the other hand, estimates from the Gaussian model implies fast mean-reversion of economic conditions and a very persistent process for the target rate.

\footnotetext{
${ }^{19}$ The stationarity of $r_{t}$ demands that $\delta_{r}<0$ and that $\left|\phi_{z}\right|<1$ (if $\delta_{z} \neq 0$ ). The stationarity of $z_{t}$ only demands the second condition.
} 


\section{Table V: Gaussian Term Structure Model}

Parameter estimates for the Gaussian term structure model. Panel (a) reports estimates of the policy rule,

$$
r_{t+1}-r_{t} \mid r_{t}, z_{t+1},\left(I_{t+1}=1\right) \sim \mathcal{D}\left(\delta_{r}\left(r_{t}-\bar{r}\right)+\delta_{z}\left(z_{t+1}-\bar{z}\right), \sigma_{1}^{2}\right),
$$

(i.e., $I_{t+1}=1$ ) where $r_{t}$ is the target rate, $z_{t}$ is a latent economic indicator, and $\mathcal{D}(\cdot, \cdot)$ is the distribution of the policy rate target innovations. It also reports the estimate of $\phi_{z}$. Panel (b) displays the estimates of the price of risk coefficients (see Equations 13 and 14). Estimation uses daily data including the target rate, the effective spread and futures rates, from Feb. 4rd, 1994 to Dec. 30th, 2011.

(a) Policy Rule

\begin{tabular}{ccccc}
\hline \hline$\sigma_{0}$ & $\sigma_{1}$ & $\delta_{r}$ & $\delta_{z}$ & $\phi_{z}$ \\
3.09 & $5 e^{-5}$ & $1.84 e-4$ & 0.023 & 0.995 \\
$(0.04)$ & $(0.04)$ & $(0.36)$ & $(0.0004)$ & $(0.0001)$ \\
\hline
\end{tabular}

(b) Prices of Risk

\begin{tabular}{cccc}
\hline \hline$\lambda_{s}$ & $\lambda_{0, z}$ & $\lambda_{z, r}$ & $\lambda_{z, z}$ \\
$8.20 e^{4}$ & $1.81 e^{3}$ & $-1.01 e^{7}$ & $-1.82 e^{6}$ \\
$\left(0.31 e^{4}\right)$ & $\left(0.15 e^{3}\right)$ & $\left(0.04 e^{7}\right)$ & $\left(0.08 e^{6}\right)$ \\
\hline
\end{tabular}

Table VI: Discrete Term Structure Model

Parameter estimates for the Skellam term structure model. Panel (a) reports estimates of the policy rule,

$$
r_{t+1}-r_{t} \mid r_{t}, z_{t+1},\left(I_{t+1}=1\right) \sim \mathcal{D}\left(\delta_{r}\left(r_{t}-\bar{r}\right)+\delta_{z}\left(z_{t+1}-\bar{z}\right), \sigma_{1}^{2}\right),
$$

where $r_{t}$ is the target rate, $z_{t}$ is a latent economic indicator, and $\mathcal{D}(\cdot, \cdot)$ is the distribution of the policy rate target innovations. It also reports the estimate of $\phi_{z}$. Panel (b) displays the estimates of the price of risk coefficients (see Equations 13 and 14). Estimation uses daily data including the target rate, the effective spread and futures rates, from Feb. 4rd, 1994 to Dec. 30th, 2011. Standard errors in parenthesis.

(a) Policy Rule

\begin{tabular}{ccccc}
\hline \hline$\sigma_{0}$ & $\sigma_{1}$ & $\delta_{r}$ & $\delta_{z}$ & $\phi_{z}$ \\
1.39 & 96.33 & -0.30 & 0.016 & 0.999 \\
$(0.10)$ & $(3.70)$ & $(0.006)$ & $(0.0002)$ & $(0.0001)$ \\
\hline
\end{tabular}

(b) Prices of Risk

\begin{tabular}{cccc}
\hline \hline$\lambda_{s}$ & $\lambda_{0, z}$ & $\lambda_{z, r}$ & $\lambda_{z, r}$ \\
$-1.54 e^{3}$ & $2.61 e^{3}$ & $-4.11 e^{7}$ & $1.73 e^{6}$ \\
$\left(0.79 e^{3}\right)$ & $\left(0.05 e^{3}\right)$ & $\left(0.51 e^{7}\right)$ & $\left(0.24 e^{6}\right)$ \\
\hline
\end{tabular}




\section{Is target rate risk priced?}

This Section relaxes the restriction that target rate innovations are not priced. Instead of Equation 13, the pricing kernel is now given by:

$$
M_{t+1}=\frac{\exp \left(\lambda_{r} \eta_{t+1}+\lambda_{s} \epsilon_{t+1}^{s}+\lambda_{t, z} \epsilon_{t+1}^{z}\right)}{E_{t}\left[\exp \left(\lambda_{r} \eta_{t+1}+\lambda_{s} \epsilon_{t+1}^{s}+\lambda_{t, z} \epsilon_{t+1}^{z}\right)\right]},
$$

where $\lambda_{r}$ is the constant price of target risk. Table VII reports pricing error RMSE and parameter estimates. Adding a single parameter increases the log-likelihood by 494 . In other words, the statistical evidence is overwhelmingly in favour of allowing target rate innovations to be priced. This addition also leads to small decreases of the pricing error RMSEs and of the standard deviation estimates.

More importantly, changes in the parameter estimates are economically significant. Introducing a constant price of target rate risk introduces risk premium variations that are associated with target rate innovations. In contrast, a constant price of risk only contributes a constant to the risk premium in the Gaussian case. The difference arises because a constant price of target risk affects all the parameters of the policy rule (not only the constant as in the Gaussian case). Fontaine (2011) shows that the intensity parameters of the Skellam distribution (see Equation 5) are shifted as follows,

$$
\begin{array}{ll}
\lambda_{u, r}^{Q}=\lambda_{r} e^{\lambda_{r} \Delta} & \lambda_{d, r}^{Q}=\lambda_{r} e^{-\lambda_{r} \Delta} \\
\lambda_{u, z}^{Q}=\lambda_{z} e^{\lambda_{r} \Delta} & \lambda_{d, z}^{Q}=\lambda_{z} e^{-\lambda_{r} \Delta} \Delta .
\end{array}
$$

where, as before, $\Delta=0.25 \%$. In particular, the loadings of $\lambda^{u}\left(r_{t}, z_{t+1}, I_{t+1}\right)$ and $\lambda^{d}\left(r_{t}, z_{t+1}, I_{t+1}\right)$ on $r_{t}$ and $z_{t+1}$ are the same under the historical probability but they are not the same under $\mathbb{Q}$. The corresponding policy rule parameters for the distribution of the target rate are given by (compare with Equation 7)

$$
\begin{aligned}
& \delta_{r}^{Q}=\left(\lambda_{u, r}^{Q}+\lambda_{d, r}^{Q}\right) \Delta=\lambda_{r}\left(e^{\lambda_{r} \Delta}+e^{-\lambda_{r} \Delta}\right) \Delta \\
& \delta_{z}^{Q}=\left(\lambda_{u, z}^{Q}+\lambda_{d, z}^{Q}\right) \Delta=\lambda_{z}\left(e^{\lambda_{r} \Delta}+e^{-\lambda_{r} \Delta}\right) \Delta
\end{aligned}
$$

The parameters $\delta_{r}$ and $\delta_{z}$ determine the policy rate dynamics under the historical measure and are used to compute the likelihood of observed policy rate changes. In contrast, the parameters $\delta_{r}^{Q}$ and $\delta_{z}^{Q}$, determine expected target changes under $\mathbb{Q}$ and are used to compute futures rates (and other asset prices). If $\lambda_{r}=0$, then these parameters are the same. Therefore, the price of target rate 


\section{Table VII: Discrete Support Model - Priced Target Risk}

Parameters estimates for the Skellam term structure model with priced target rate risk. Panel (a) reports pricing RMSE for each maturity and the corresponding estimated standard deviation parameters. Panel (a) reports estimates of the policy rule, $\sigma_{0}$ and

$$
r_{t+1}-r_{t} \mid r_{t}, z_{t+1},\left(I_{t+1}=1\right) \sim \mathcal{D}\left(\delta_{r}\left(r_{t}-\bar{r}\right)+\delta_{z}\left(z_{t+1}-\bar{z}\right), \sigma_{1}^{2}\right),
$$

(i.e., $I_{t+1}=1$ ) where $r_{t}$ is the target rate, $z_{t}$ is a latent economic indicator, and $\mathcal{D}(\cdot, \cdot)$ is the distribution of the policy rate target innovations. It also reports the estimate of $\phi_{z}$. Panel (b) displays the estimates of the price of risk coefficients (see Equations 13 and 14) including the estimate of the (constant) price of target rate risk. Estimation uses daily data including the target rate, the effective spread and futures rates, from Feb. 4rd, 1994 to Dec. 30th, 2011. Standard errors in parenthesis.

(a) Pricing Errors

\begin{tabular}{ccccccc}
\hline \hline & \multicolumn{7}{c}{ Maturity } \\
\cline { 2 - 7 } & 1 & 2 & 3 & 4 & 5 & 6 \\
RMSE & 4.36 & 6.57 & 4.87 & 3.49 & 2.25 & 4.01 \\
$\xi(n)$ & 5.22 & 4.89 & 4.56 & 4.24 & 3.91 & 3.59 \\
\hline
\end{tabular}

(b) Policy Rule

\begin{tabular}{ccccc}
\hline \hline$\sigma_{0}$ & $\sigma_{1}$ & $\delta_{r}$ & $\delta_{z}$ & $\phi_{z}$ \\
0.68 & 20.98 & -0.07 & 0.0032 & 0.999 \\
$(0.03)$ & $(1.05)$ & $(0.004)$ & $(0.0002)$ & $(0.0002)$ \\
\hline
\end{tabular}

(c) Prices of Risk

\begin{tabular}{ccccc}
\hline \hline$\lambda_{r}$ & $\lambda_{s}$ & $\lambda_{0, z}$ & $\lambda_{z, r}$ & $\lambda_{z, z}$ \\
$3.27 e^{5}$ & $-1.62 e^{3}$ & $6.76 e^{3}$ & $-7.06 e^{7}$ & $3.04 e^{6}$ \\
$\left(0.09 e^{5}\right)$ & $\left(0.08 e^{3}\right)$ & $\left(0.39 e^{3}\right)$ & $\left(0.21 e^{7}\right)$ & $\left(0.24 e^{6}\right)$ \\
\hline
\end{tabular}


risk introduces a time-varying risk premium in the dynamics of the target rate,

$$
E_{t}^{Q}\left[r_{t+1}-r_{t}\right]-E_{t}\left[r_{t+1}-r_{t}\right]=\left(\delta_{r}^{Q}-\delta_{r}\right)\left(r_{t}-\bar{r}\right)+\left(\delta_{z}^{Q}-\delta_{z}\right) E_{t}\left[z_{t+1}\right]+\delta_{z}^{Q}\left(E_{t}^{Q}\left[z_{t+1}\right]-E_{t}\left[z_{t+1}\right]\right)
$$

where the last term is due to variation in the price of macro risk (see below). The estimate of the price of target risk is positive and significant. Unexpected increases in the target rate are considered risky - they are associated with bad states of the economy from the point view of participants in the futures markets. The implied parameter shift is substantial $-e^{\hat{\lambda}_{r} \Delta}+e^{-\hat{\lambda}_{r} \Delta}=9.79$. Under the historical measure, the policy rule coefficient estimates are $\hat{\delta}_{r}=-0.07$ and $\hat{\delta}_{z}=0.0032$. Both are significantly lower than the previous estimates. However, under the risk-neutral measure, the corresponding parameters are $\hat{\delta}_{r}^{Q}=-0.33$ and $\hat{\delta}_{z}^{Q}=0.015$. These are almost identical to the estimates of $\delta_{r}$ and $\delta_{z}$ above (where $\delta^{Q}=\delta$ ). Hence, allowing for the price of target risk makes the expected target rate less sensitive to economic conditions and with less mean-reversion under $\mathbb{P}$

than under $\mathbb{Q}$. This implies that the risk premium for target rate risk, $E_{t}^{Q}\left[r_{t+1}-r_{t}\right]-E_{t}\left[r_{t+1}-r_{t}\right]$, is higher when economic conditions improve and when the target rate is high.

\section{The market price of macro risk}

Figure 4 displays estimates of $\lambda_{z, t}$, the parameter controlling the time-varying price of risk associated with innovations in the macro factor. The correlations between the two series is significant : 0.84 . However, there are important differences between the two models. The price of risk varies smoothly, and mostly at the monthly frequency. Moreover, while the price of risk almost settled around a constant values in the discrete model since the target rate reached its lower bound. In contrast, estimates from the Gaussian model still vary significantly. Also, the price of macro risk is typically positive in the Discrete model. Then, estimates of the price of macro risk from the discrete model are consistent with an established view that the risk premium implicit in futures consistent exhibit little variations at the daily frequency.

The results are also consistent with the fact that innovations in the macro factors should be associated with lower marginal rate of substitution and improved economic conditions for investors. In contrast, the estimates of price of risk from the Gaussian model exhibits significant daily variations and sometimes swing deeply in the negative territory. Moreover, the distortions is significant for policy purposes. Estimates of the price of risk in the Gaussian model reach large negative values when the target rate declines faster than expected, or when the target rate is close to its lower bound. Therefore, large negative risk premium imply estimates target rates expectations that are biased upward in periods where the Fed's intent is to loosen monetary conditions. Overall, the 
evidence suggests that the mis-specification of the target rate distribution induces economically significant bias in the estimates of the prices of macro risk.

\section{Unconventional Policy and the Challenges Ahead}

This Section shows that the filtered values of the latent macro factor can be interpreted as changes in economic conditions implicit in Fed Funds futures rates. I then use the macro factor to evaluate the effect of different policy announcements. I conclude with a discussion of recent modeling efforts to address the challenges raised by unconventional policy at the zero lower bound.

\section{A Link with Economic Conditions}

The Philadelphia Fed publishes the Aruoba-Diebold-Scotti [ADS] index of U.S. real activity. The index summarizes the information content from releases of real economic variables. It does not contain information from asset prices or other nominal variables. The index is daily and is "designed to track real business conditions at high frequency. ${ }^{20}$ The underlying economic variables blend highand low-frequency information and stock and flow data (weekly initial jobless claims; monthly payroll employment, industrial production, personal income less transfer payments, manufacturing and trade sales; and quarterly real GDP)".

Figure 3 draw the correlations between current ADS index and the lagged macro factor, with daily lags from zero up to six months. It also draws the converse - the correlations between current macro factor and the lagged index. The highest lag corresponds to the longest futures maturity used to filter the macro factor. The results show that the predictive content of the macro factor for future values ADS index is substantial. The correlations increase smoothly from 0.4 at low horizons to nearly 0.6 at horizons around six months. In contrast, the predictive content of the ADS index decays smoothly to just above 0.1 .

Figure 5 compares the filtered values of the latent factor, $z_{t}$ with the ADS index. The relationship between $z_{t}$ and the index is visually apparent. These two proxies for economic conditions are closely related. Of course, they do not overlap since futures rates combine information from a broader information set perceived by market participants to be relevant for FOMC decisions. High realization of the macro factor are associated with high values of the ADS index in the future. Figure 5 also reveals that the relationship between economic activity, as measure by the ADS index, and the macro factor, measured from futures rates changes toward the end of 2008. Figure 6 provides a close-up of this period. In fact, the macro factor appears to be bounded from below.

\footnotetext{
${ }^{20} \mathrm{~A}$ similar high-frequency index of inflation is not available.
} 
This is a reflection of the Fed's decision, in December 2008, to lower its target to $0.25 \%$, which is effectively the lower bound. The ADS index continued to deteriorate in the recession of 2009 but rates could not be lowered further. The Fed's unobserved desired effect on the economy corresponds to a negative policy rate but this cannot be implemented in practice.

On the other hand, the futures curve still measures improvements of economic conditions. On June 9th 2009, the US Labor Department reported a lower number of job losses than what was expected. This corresponds to the largest spike in the macro factor in Figure 7. Nonetheless, persistently low inflation eventually led the Fed to implement quantitative easing policy and, in addition, to implement a commitment to keep the target rate unchanged until the end of 2013. There is no lower bound in a linear model. Therefore, implementation of a linear filter through a time series of a futures rates that remains relatively unchanged leads to relatively unchanged estimates of the macro factor.

\section{$B$ Policy Announcement at the Lower Bound}

We can use the macro factor to measure the of impact of policy announcements on the expectations of participants in the futures market. Table VIII lists major monetary policy announcements by the Federal Reserve since November 2008. It builds from and extends the list in Wright (2012) until the end of 2011. It includes the date of every FOMC meetings, key speeches by Fed Chairman Bernanke, and one coordinated announcement by different central banks. We can measure the effect of an announcement on the state of economy, as perceived from market participants, from changes in futures rates. The maintained assumption is that these policy announcements are the main drivers of futures rate changes (on the announcement day).

Figure 7 draws the evolution of the macro factor since 2008 until the end of 2011 with vertical lines that indicate the announcement dates listed in Table VIII. Almost every policy announcement corresponds to a decline in the macro factor. This is consistent with the Federal Reserve signalling that the target rate would remain constant for a longer horizon. This arose either because the Fed's statement related directly to the horizon, or indirectly, as a consequence of other policy decisions (e.g., quantitative easing). These declines in the macro factor are large for this period. The mean response is close in magnitude to the standard deviation of all shocks in this period. However, the shocks show little persistence and the cumulative change of the macro factor is small. This reflects the tension between the effect of the Fed's statement on long horizon futures and the lack of variations implicit in the one-month contract.

The asymmetry in the response of futures rates implies that the usefulness of a linear model is also asymmetric. Notwithstanding episodic improvements in economic conditions, the filtered 
Table VIII: Policy Announcements 2008-2011

Dates of major policy announcements by the Federal Reserve between November 2008 and December 2011. Dates until August 2009 are from Wright (2012).

\begin{tabular}{lc}
\hline \hline Date & Nature of the Announcement \\
\hline $11 / 25 / 2008$ & Fed Announces Purchases of MBS and Agency Bonds \\
$12 / 1 / 2008$ & Bernanke states Treasuries may be purchased \\
$12 / 16 / 2008$ & Scheduled FOMC Meeting \\
$1 / 28 / 2009$ & Scheduled FOMC Meeting \\
$3 / 18 / 2009$ & Scheduled FOMC Meeting \\
$4 / 29 / 2009$ & Scheduled FOMC Meeting \\
$6 / 24 / 2009$ & Scheduled FOMC Meeting \\
$8 / 12 / 2009$ & Scheduled FOMC Meeting \\
$9 / 23 / 2009$ & Scheduled FOMC Meeting \\
$11 / 4 / 2009$ & Scheduled FOMC Meeting \\
$12 / 16 / 2009$ & Scheduled FOMC Meeting \\
$1 / 27 / 2010$ & Scheduled FOMC Meeting \\
$3 / 16 / 2010$ & Scheduled FOMC Meeting \\
$4 / 28 / 2010$ & Scheduled FOMC Meeting \\
$6 / 23 / 2010$ & Scheduled FOMC Meeting \\
$8 / 10 / 2010$ & Scheduled FOMC Meeting \\
$8 / 27 / 2010$ & Bernanke Speech at Jackson Hole \\
$9 / 21 / 2010$ & Scheduled FOMC Meeting \\
$10 / 15 / 2010$ & Bernanke Speech at Boston Fed \\
$11 / 3 / 2010$ & Scheduled FOMC Meeting \\
$12 / 14 / 2010$ & Scheduled FOMC Meeting \\
$1 / 26 / 2011$ & Scheduled FOMC Meeting \\
$3 / 15 / 2011$ & Scheduled FOMC Meeting \\
$4 / 27 / 2011$ & Scheduled FOMC Meeting \\
$6 / 2 / 2011$ & Scheduled FOMC Meeting \\
$8 / 9 / 2011$ & Scheduled FOMC Meeting \\
$8 / 26 / 2011$ & Bernanke Speech at Jackson Hole \\
$9 / 21 / 2011$ & Scheduled FOMC Meeting \\
$11 / 2 / 2011$ & Scheduled FOMC Meeting \\
$11 / 30 / 2011$ & Scheduled FOMC Meeting \\
$12 / 13 / 2011$ & \\
\hline &
\end{tabular}


macro factor indicated throughout 2010 and 2011 that these did not lead market participants to revise their expectations of policy rates upward. On the other hand, the futures rates were relatively insensitive to further deterioration of the underlying economic conditions. It is true that policy announcements by the FOMC still affected the futures market and led to declines in the macro factor. Nonetheless, this measured effect was low by historical standard given that the target rate is bounded below.

\section{The Challenges Ahead}

The lower bound on nominal interest rates is the current frontier for policy-makers and researchers studying the term structure of interest rates. Several alternative solutions exist in the literature. First, the seminal work of Cox et al. (1985) is based on "square-root" processes and maintains the positivity of yields as well as computational tractability. But note that most existing empirical applications enforce the Feller conditions. This approach is often criticized on the grounds that zero becomes a reflecting boundary - the short rate bounces off zero and never reaches it. Following this observation, Black (1995) formulated the short rate as an option,

$$
r_{t}=\max \left(0, \tilde{r}_{t}\right)
$$

where $\tilde{r}_{t}$ is the "shadow" policy rate. This allows for more flexibility in the specification of $\tilde{r}_{t}$ but the analyst must resort to numerical solutions for yields and other asset prices. Finally, a quadratic short rate is always positive,

$$
r_{t}=x_{t}^{\prime} \Upsilon x_{t}
$$

and analytical results are available when $x_{t}$ is Gaussian. This leads to quadratic-Gaussian term structure models (Ahn et al. 2002 and Leippold and Wu (2003)) where the solution for yields is a linear-quadratic function of $x_{t}$.

Kim and Singleton (2011) provide an empirical study of nominal yields in Japan between 1995 and 2008. They show that the behaviour of yields is qualitatively different in this period. Empirically, they compare multi-factor square-root, shadow-rate and quadratic-Gaussian term structure models. They focus on long-maturity yields (the shortest maturity in their sample is 6 months) and find that the shadow-rate and quadratic-Gaussian models capture important stylized features of long-term interest rates in Japan. The same is not true for the square-root model.

These formulations are targeted at yields with relatively long maturity and toward relatively low sampling frequencies. They ignore the fact that changes in the target rate are lumpy and occur only rarely. As evidenced above, this omission affects estimation results if one focuses on yields with 
short maturities at a relatively high frequency. Hence, the pervasive, and sometimes persistent, occurrences of very low policy rates nowadays calls for the development of term structure models that meet the empirical features of the target rate while also enforcing the positivity of yields.

Some recent work seeks to reach this goal. Feunou and Fontaine (2012) introduce the family of Discrete Choice Dynamics Term Structure models. They define the policy response function as an ordered discrete choice problem (McFadden, 1984) and obtains asset pricing results to develop a model of the term structure of yields. These models are flexible and allow for rich non-linearities in the relationship between yields and the underlying economic variables, including the zero bound. An ordered choice representation of the policy rule has been proposed before (Hamilton and Jordà, 2002) but implications for the term structure of yields have been ignored. Renne (2012) provides an alternative specification based on regime-switching techniques. Although the number of regimes is

large, this family of models leads to a tractable affine representation of yields. Empirically, Renne (2012) applies this model to the Euro area in a daily sample between 1999 and 2012. Finally, the approach in Black (1995) is also applicable if the shadow-rate has a discrete support, as in this paper. But the model then becomes intractable.

\section{Conclusion}

This chapter shows that different modeling assumptions yield different interpretations of the information embedded in the term structure of yields. It provides a specification of the term structure that is consistent with the operational policy of modern central banks. Policy rate changes occur infrequently, typically on scheduled dates, and they are lumpy (i.e., multiples of $0.25 \%$ ). The target rate in the US has been fixed to its lower bound since the end of 2008. The positivity constraint has been addressed in the literature in the context of long-term maturities but still poses a challenge when combined with the requirement that the target rate has a discrete support. Moreover, the lower bound does not imply that the central bank takes no further action to meet its mandates. Liquidity facilities, credit easing, quantitative easing, and others, all represent monetary policy actions unrelated to the conventional target rate but that affect the term structure of interest rates. Understanding the transmission of these unconventional actions to long-term yields is the next challenge that the next generation of models must meet. 


\section{References}

Ahn, D.-H., R. Dittmar, and A. R. Gallant (2002). Quadratic term structure models : theory and evidence. Review of Financial Studies 15, 243-288.

Balduzzi, P., G. Bertola, and S. Foresi (1997). A model of target changes and the term structure of interest rates. Journal of Monetary Economics 39, 223-249.

Bech, M. L. and E. Klee (2009). The mechanics of a graceful exit: Interest on reserves and segmentation in the federal funds market. Working paper 416, Federal Reserve Bank of New York.

Bhundia, A. J. and J. S. Chadha (1998). The information content of 3-month sterling futures. Economics Letters 61(2), $209-214$.

Black, F. (1995). Interest rates as options. The Journal of Finance 50, 1371-1376.

Clarida, R., J. Galí, and M. Gertler (2000). Monetary policy rules and macroeconomic stability : Evidence and some theory. The Quarterly Journal of Economics 115, 147-180.

Cochrane, J. and M. Piazzesi (2002). The Fed and interest rates : A high-frequency identification. American Economic Review 92, Papers and Proceedings, 90-95.

Cox, J., J. Ingersoll, and S. Ross (1985). A theory of the term structure of interest rates. Econometrica 53, 385-407.

Diez de los Rios, A. and C. Reid (2008). Extracting policy rate expectations in canada.

Duffee, G. (2002). Term premia and interest rate forecasts in affine model. The Journal of Finance 57, 405-443.

Duffee, G. (2009). Sharpe ratios in term structure models. Working paper, Johns Hopskins University.

Duffee, G. R. (1996). Idiosyncratic variation of Treasury bill yields. The Journal of Finance 51(2), 527-551.

Feldhtter, P. and D. Lando (2008). Decomposing swap spreads. Journal of Financial Economics 88(2), 375 $-405$.

Ferrero, G. and A. Nobili (2008). Futures contract as monetary policy forecasts. Technical Report 979, European Central Bank.

Feunou, B. and J. Fontaine (2012). Discrete-choice term structure models : Theory and applications. Banque du Canada.

Fontaine, J.-S. (2011). Fed funds futures and the federal reserve. Working Paper.

Gurkaynak, R., B. Sack, and E. Swanson (2007). Market based measures of monetary policy expectations. Journal of Business and Economics Statistics 25, 201-212.

Hamilton, J. (1996). The daily market for federal funds. The Journal of Political Economy 104, 26-56.

Hamilton, J. (2009). Daily changes in Fed funds futures prices. Journal of Money, Credit and Banking 41, $567-582$. 
Hamilton, J. and O. Jordà (2002). A model of the federal funds rate target. The Journal of Political Economy 110, 1136-1167.

Hamilton, J. and T. Okimoto (2010). Sources of variations in holding returns for Fed funds futures contracts. Journal of Futures Markets. forthcoming.

Hamilton, J. D. (1997). Measuring the liquidity effect. The American Economic Review 87(1), 80-97.

Kim, D. H. and K. Singleton (2011). Term structure models and the zero bound: An empirical investigation of japanese yields.

Krueger, J. and K. Kuttner (1996). The Fed funds futures rate as a predictor of Federal Reserve policy. International Economic Review 16, 865-879.

Leippold, M. and L. Wu (2003). Design and estimation of multi-currency quadratic models. European Finance Review 7, 47-73.

McFadden, D. L. (1984). Handbook of Econometrics, Chapter Econometric analysis of qualitative response models. Elsevier.

Piazzesi, M. (2005a). Bond yields and the Federal Reserve. The Journal of Political Economy 113, 311-344.

Piazzesi, M. (2005b). Handbook of Financial Econometrics, Chapter "Affine term structure models". Elsevier.

Piazzesi, M. and E. Swanson (2006). Futures prices as risk-adjusted forecasts of monetary policy. Technical Report 2006-23, Federal Reserve Bank of San Francisco.

Renne, J.-P. (2012). A model of the euro-area yield curve with discrete policy rates. Technical report, Banque de France. working paper.

Rigobon, R. (2003). Identification through heteroskedasticity. Review of Economics and Statistics 85(4), $777-792$.

Rudebusch, G. (1995). Federal reserve interest rate targeting, rational expectations, and the term structure. Journal of Monetary Economics 35(2), 245 - 274.

Sack, B. (2004). Extracting the expected path of monetary policy from futures rates. International Economic Review 24, 733-754.

Sentana, E. and G. Fiorentini (2001). Identification, estimation and testing of conditionally heteroskedastic factor models. Journal of Econometrics 102(2), 143 - 164.

Sin, C. and H. White (1996). Information criteria for selecting possibly misspecified parametric models. Journal of Econometrics 71(12), 207 - 225.

Skellam, J. (1946). The frequency distribution of the difference between two Poisson variates belonging to different populations. Journal of the Royal Statistical Society 109, 296.

Taylor, J. B. (1993). Discretion versus policy rules in practice. Carnegie-Rochester Conference Series on Public Policy 39, $195-214$.

Thornton, D. L. (2006). The daily liquidity effect.

Wright, J. (2012). What does monetary policy do to long-term interest rates at the zero lower bound? 


\section{Appendix}

\section{A Discrete Changes and the Distribution of Yields}

Long-term yields evolve smoothly because the expectation operator is not restricted to the support of any random variable. ${ }^{21}$ To see this, define the set of future dates where a meeting is scheduled, $\mathbb{S}_{t}$, and write the one-year yield, $y_{t}^{(i)}$, in terms of target rate expectations and a risk premium,

$$
y_{t}^{(1)}=E_{t}\left[\frac{1}{365} \sum_{i=1}^{365} r_{t+i-1}\right]+r p_{t}^{(1)}=r_{t}+E_{t}\left[\sum_{i \in \mathbb{S}_{t}} c_{i} \Delta_{t+i}\right]+r p_{t}^{(1)}
$$

where time is daily, $\Delta_{t+i}=r_{t+i}-r_{t+i-1}, c_{i}=\frac{365-i}{365}$ and $r p_{t}^{(1)}$ is an adjustment for risk. Then, on days with no policy rate changes (i.e., $r_{t}=r_{t+1}$ ), yield changes are given by,

$$
y_{t+1}^{(1)}-y_{t}^{(1)}=\sum_{i \in \mathbb{S}}\left(E_{t+1}-E_{t}\right)\left[c_{i} \Delta_{t+i}\right]+r p_{t+1}^{(1)}-r p_{t}^{(1)},
$$

which shows that yields can change smoothly as economic information arrives since expectations and, a fortiori, expectation changes are not restricted to the discrete support. Moreover, yields average the expected changes over a sequence of future meetings. Finally, risk premium variations can also evolve continuously.

But discrete target changes affect the distribution of yields changes. Note that the first term in Equation 24 is different on days where a policy meeting is held. On non-meeting days, this term is the change in the expectations of future target rate. On meeting days, it is the policy rate innovation, $n_{t+1}=E_{t}\left[r_{t+1}-r_{t}\right]-\left(r_{t+1}-r_{t}\right)$ (with a slight abuse of notation). Changes in expectations summarize the effect of a variety of "news" related to future policy and may be relatively close to a Gaussian distribution. In contrast, the innovations, $n_{t+1}$, can take only a few values ex-post. In general, the expectation term, $E_{t}\left[r_{t+1}\right]$, does not lie close to a point on the support of $r_{t+1}$ and the policy innovation $n_{t+1}$ will "jump" to one of these points. Large yield changes should be more common on days with policy meetings.

To confirm this effect, I consider Federal Funds futures contracts with maturities between 1 and 6 months. I count occurrences of rate changes in bins with different change sizes on days with a scheduled policy meeting. The edges of the bins are $-7.5,-2.5,2.5$ and $7.5 \mathrm{bps}^{22} \mathrm{I}$ consider each maturity separately. The sample runs from 1994 until 2011 and covers 144 schedule meetings. I repeat the analysis for other days when no meeting occurs. Table IX reports for each bin the ratio of counts on meeting days to the average of counts across the preceding 20 non-meeting days. For each maturity, and for each bin, a ratio lower than one indicates that changes are less frequent on meeting days and on non-meeting days. ${ }^{23}$

As expected, small changes are less likely on meeting days. Overall, changes between -2.5 bps and 2.5 bps were between $15 \%$ and $25 \%$ less frequent on meeting days than on non-meeting days. In contrast, larger rate changes are more frequent. Changes between 2.5 and 7.5 bps (in absolute value) are typically $50 \%$ more frequent on meeting days. The difference is much greater for the 1-month contract: there are very few changes of this magnitude on non-meeting days at this maturity. Finally, rate changes greater than 7.5 bps (in absolute value) are typically twice as likely on meeting days than on non-meeting days. Therefore,

\footnotetext{
${ }^{21}$ But it is restricted to its range

${ }^{22}$ For comparison, the standard deviation of rate changes for the 6 -month contract is 4.6 bps.

${ }^{23}$ See Section A for a description of Federal Funds futures contracts. Note that the results are robust to changes in the size of the bins and to changes in the number of days used to compute the average counts on non-meeting days.
} 


\section{Table IX: Futures Rates Changes}

Relative frequency of futures rate changes on days with and without policy meetings. Each line corresponds to a different contract maturity. I report the ratio of the number of times a futures rate changes on days with or without policy meeting, $P\left(x_{i} \leq f_{t, n}-f_{t-1, n} \leq x_{i+1} \mid I_{t}=1\right)$ and $P\left(x_{i} \leq f_{t, n}-f_{t-1, n} \leq x_{i+1} \mid I_{t}=0\right)$, respectively, where $f_{t}$ is the rate on the $n$-month ahead contract, $I_{t}$ is an indicator function equal to 1 when a meeting occurs, and $x_{i}$ are the edges of the different bins used to compute the probabilities. $P$ is the empirical distribution. Daily data from Jan. 3rd, 1994 to Dec. 30th, 2011. Contract maturities between 1 and 6 months ahead.

\begin{tabular}{crrrrrrr}
\hline \hline & \multicolumn{7}{c}{ Maturity } \\
$f_{t, n}-f_{t-1, n}$ & & 1 & 2 & 3 & 4 & 5 & 6 \\
\cline { 1 - 4 }$-\infty \leq-0.075$ & & 1.36 & 4.38 & 2.92 & 2.89 & 2.63 & 2.31 \\
$-0.075 \leq-0.025$ & & 5.74 & 1.41 & 1.66 & 1.43 & 1.41 & 1.28 \\
$-0.025 \leq 0.025$ & & 0.84 & 0.80 & 0.83 & 0.81 & 0.76 & 0.80 \\
$0.025 \leq 0.075$ & & 4.42 & 1.84 & 1.38 & 1.38 & 1.15 & 1.04 \\
$0.075 \leq \infty$ & 1.54 & 5.00 & 2.43 & 2.05 & 2.75 & 2.05 \\
\hline
\end{tabular}

discrete changes in the target rate imply that yield changes have a more dispersed distribution on following policy announcements. 


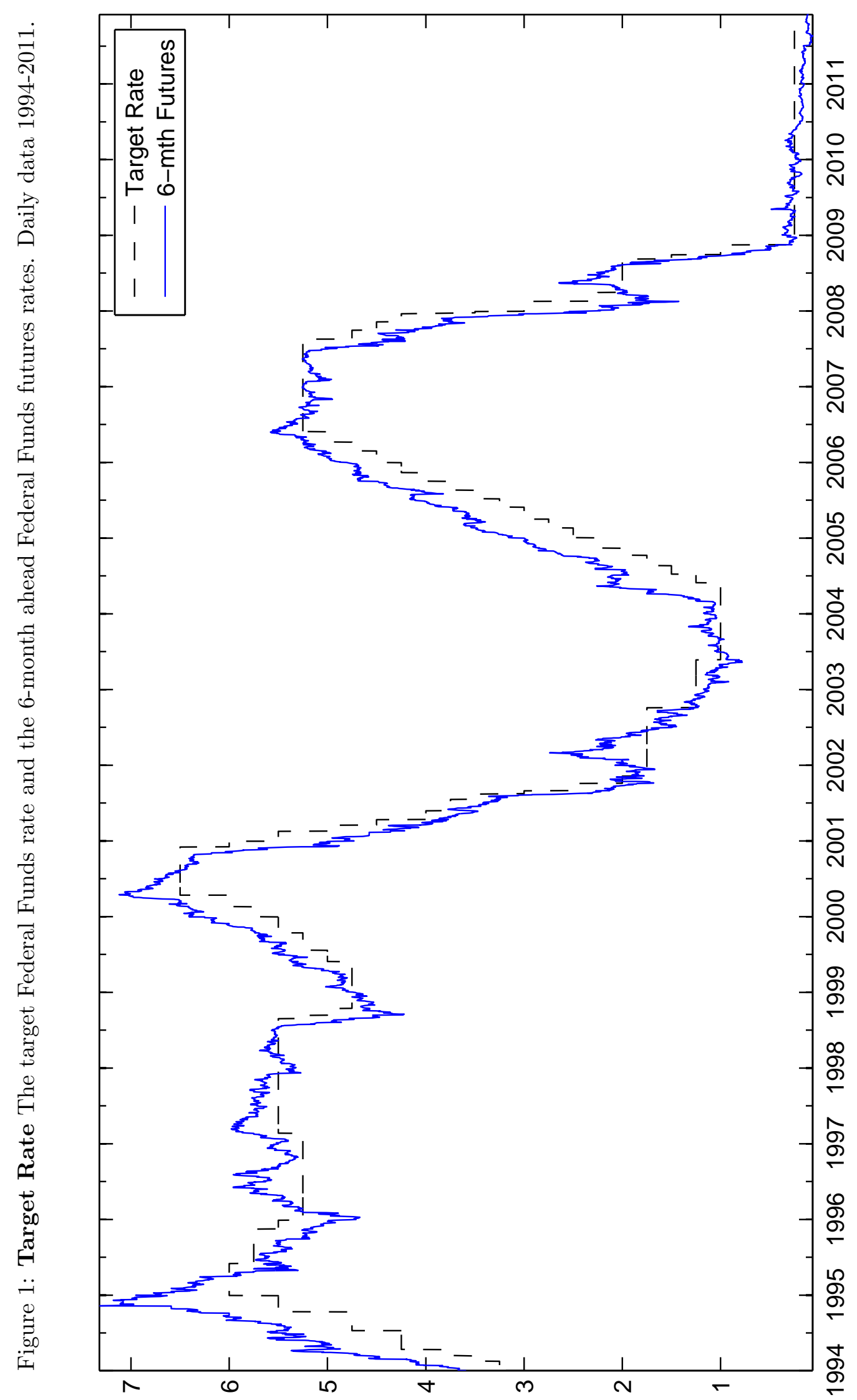




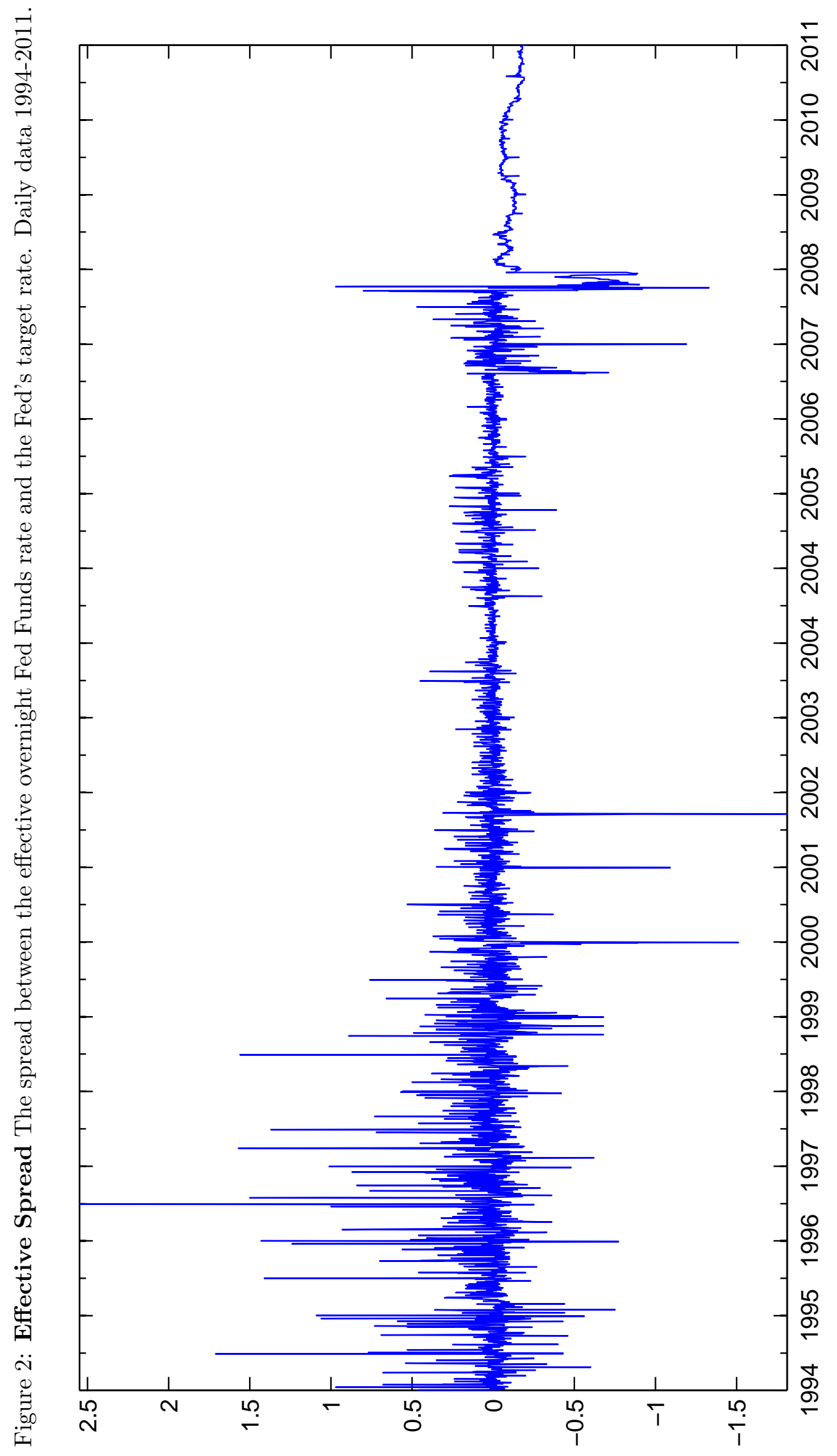




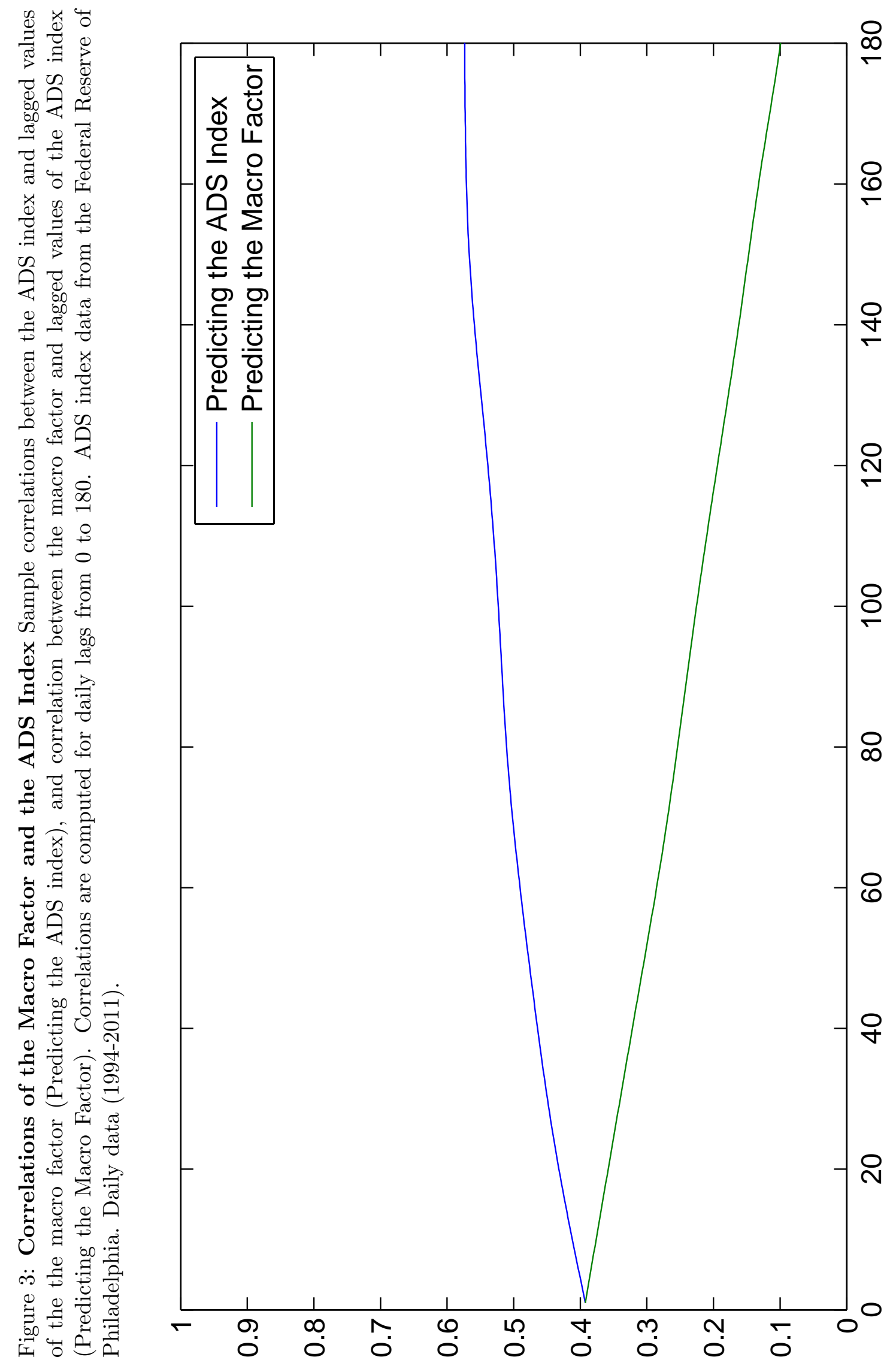




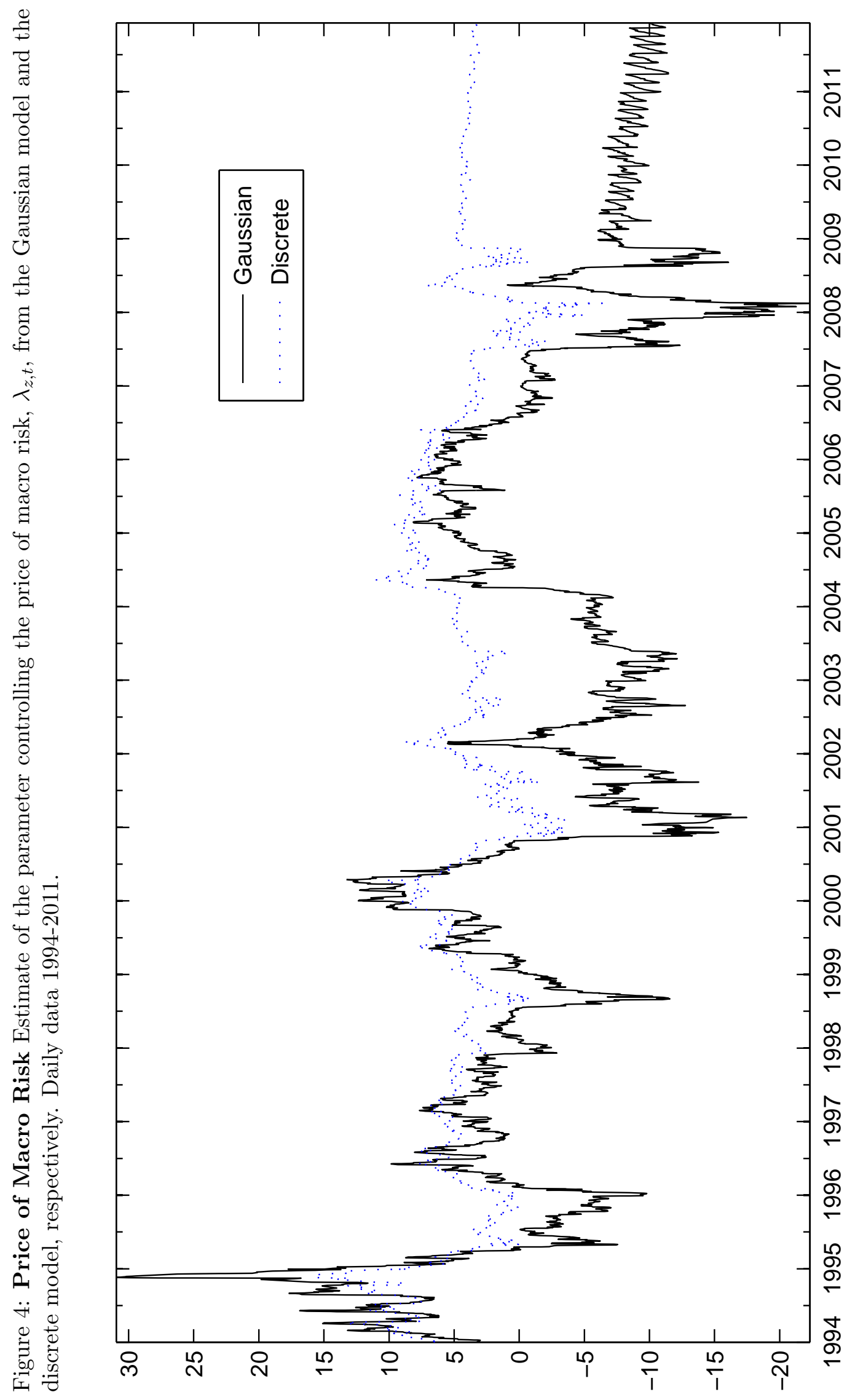




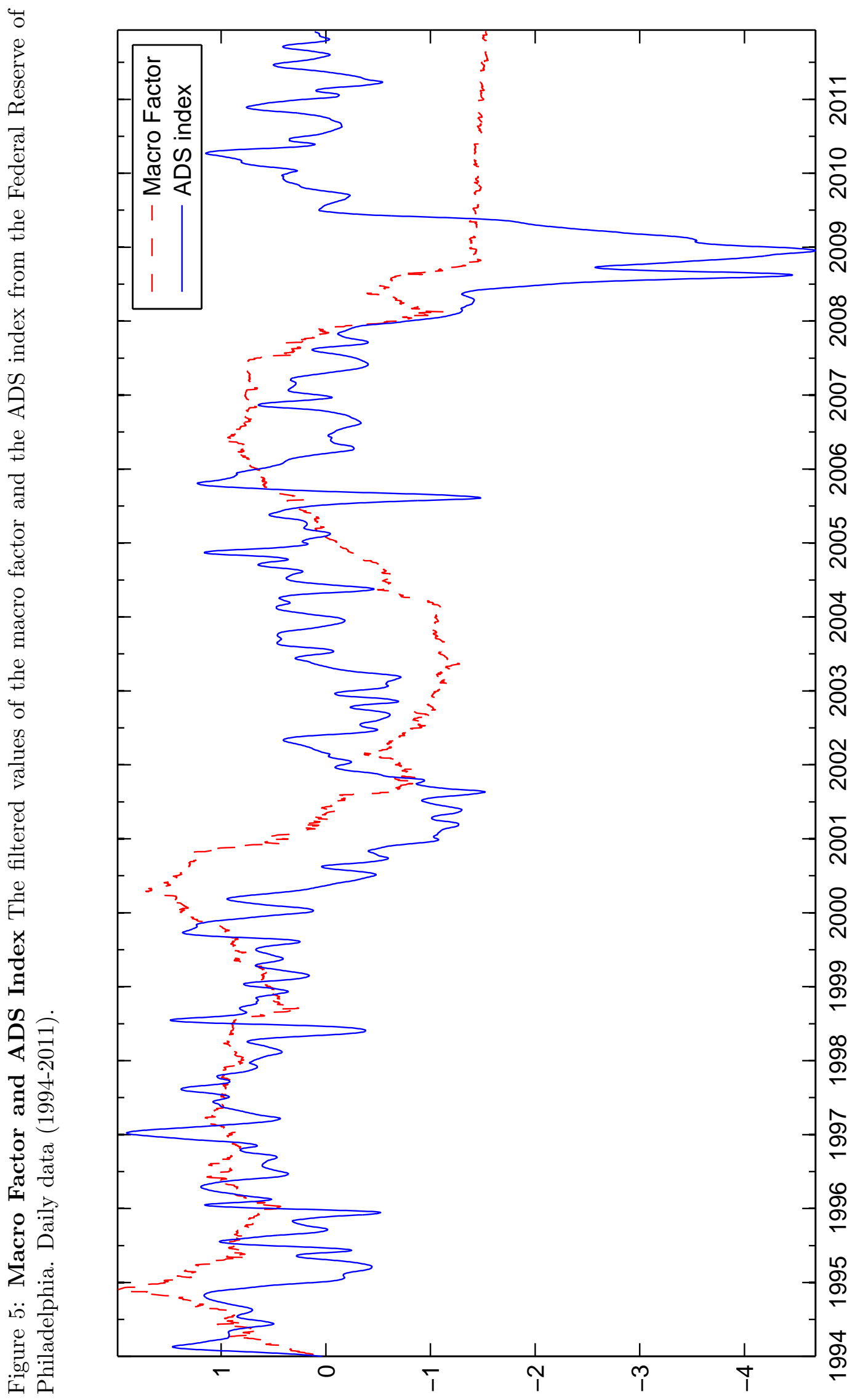




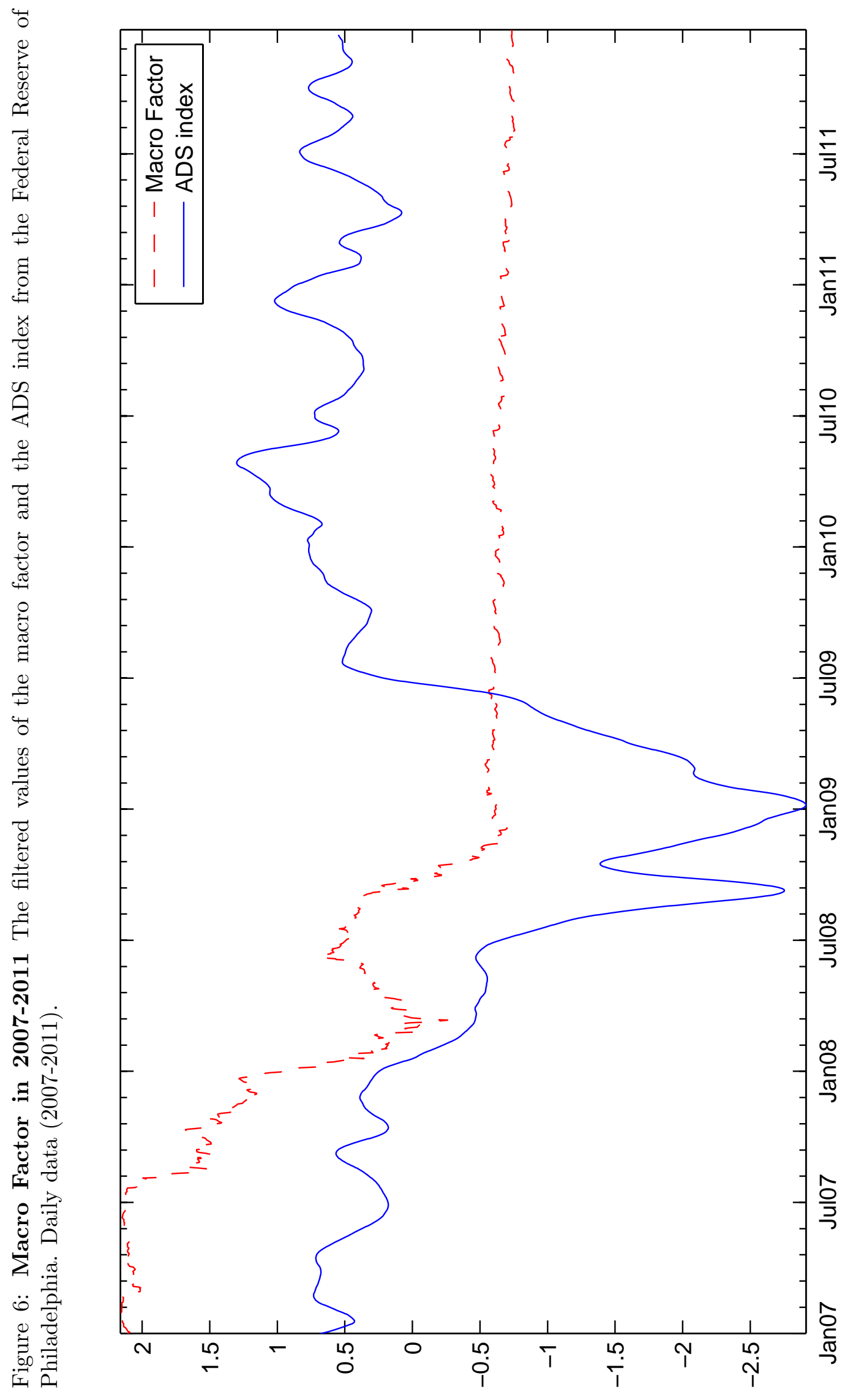




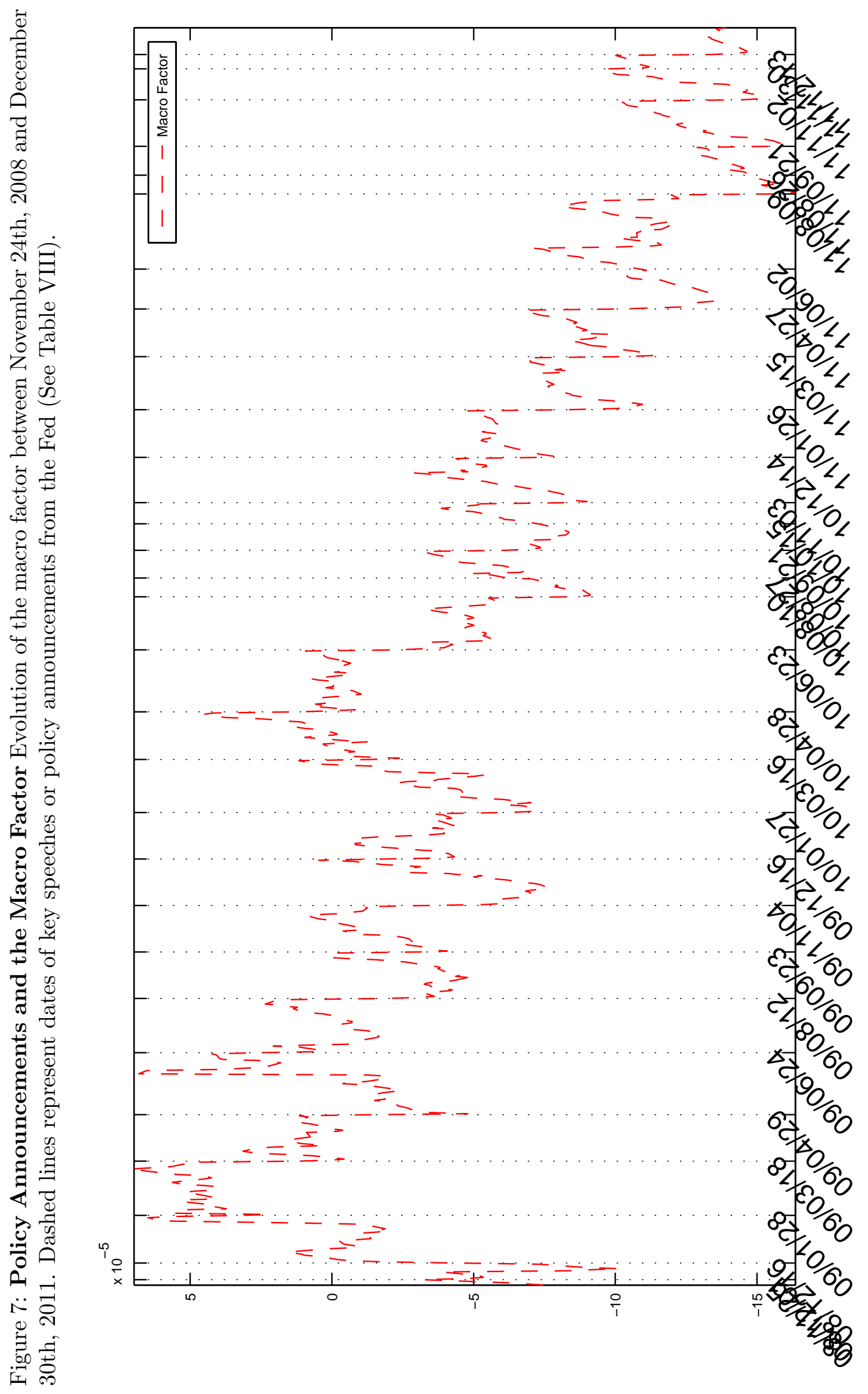

